\title{
Producción y descomposición de biomasa seca y transferencia de nutrientes en sistemas agroforestales con café
}

Fernando Farfán Valencia, Ingeniero Agrónomo Investigador, Centro Nacional de Investigaciones de Café - Cenicafé.

\section{INTRODUCCIÓN}

El componente arbóreo en un sistema agroforestal busca optimizar el uso de recursos y aumentar la productividad por unidad de terreno, además de ser fuente de energía, madera, frutos y/o sombrío, puede regular las condiciones de luz para el cultivo principal y suplir parte de los nutrientes requeridos por él; así mismo, con una densidad suficiente de árboles se logran reducir los vientos fuertes, lo que contribuye a la protección del suelo y del café (74). Los árboles también aportan gran cantidad de residuos vegetales que actúan como material de cobertura (37); la capa de hojarasca es el eslabón que mantiene unidos los componentes arbóreos con el suelo, esta capa a su vez, por procesos de descomposición y mineralización, liberará nutrientes que podrán ser nuevamente absorbidos por las plantas $(59,66)$.

El retorno anual de la materia orgánica y bioelementos al suelo a través de la hojarasca, es uno de los condicionantes más importantes en la renovación del ecosistema agroforestal. El efecto que la acumulación de los restos orgánicos en la superficie del suelo tiene sobre la productividad, ha inducido a muchos autores, al estudio de la cinética de la descomposición de la hojarasca $(5,13)$. El posible papel de las especies arbóreas sobre la circulación de nutrientes en su ecosistema, depende de la cantidad de material reciclable y de su tasa de descomposición. Por ello, es importante la identificación de las especies con influencia positiva sobre la restauración de la fertilidad del suelo, para utilizarlas en el diseño de plantaciones arbóreas mixtas y sistemas agroforestales $(34,44,74)$.

En diversas partes del mundo es evidente una gran variedad de modalidades del cultivo del café, en tanto que las condiciones agroecológicas que prevalecen determinan esta modalidad. Existen fundamentalmente dos tipos de sistemas de cultivo, monocultivo y sistemas agroforestales. En Colombia, es común el cultivo del café bajo árboles de sombrío o en sistemas agroforestales, definidos como asociaciones deliberadas o sistemáticas de árboles con cultivos en el mismo espacio (16, 17, 29, 37, 67); en este artículo se presentan los resultados de las investigaciones realizadas por Cenicafé, en dos localidades 
de la zona cafetera central y sur de Colombia, en las cuales se evaluó el aporte y descomposición de la hojarasca como material vegetal, y la transferencia de nutrientes que este material realiza al suelo en sistemas agroforestales con café.

\section{-Aporte y descomposición de la hojarasca y liberación de nutrientes de Coffea arabica, Cordia alliodora, Pinus oocarpa y Eucalyptus grandis.}

Descripción del sitio de estudio. El estudio se localizó en la Subestación Experimental Paraguaicito, situada en el municipio de Buenavista (Quindío). La ubicación geográfica, clima $(9,10,11)$ y las características de suelos (19) se presentan en la Tabla 1.

Para el estudio fueron consideradas dos fases. La primera, comprendida entre agosto de 1997 y julio de 1998 y noviembre de 2000 y octubre de 2001, durante la cual se evaluó el aporte de materia seca producida por el café a libre exposición solar, el café bajo sombrío y la biomasa seca producida por tres especies forestales que dieron sombra al café. La segunda fase comprendida entre agosto de 2001 y julio de 2002, período en el cual se evaluó la tasa de descomposición de residuos vegetales y la transferencia de nutrientes; en este segundo período en La Subestación Experimental se registró una humedad relativa del $85,0 \%$, precipitación de 2.269 mm.año-1 y un promedio de temperatura de $17,0^{\circ} \mathrm{C}(9)$.

En la Figura 1, se presentan los Índices de Humedad del Suelo (IHS) calculados para la Subestación Experimental Paraguaicto para los tres períodos de estudio. El IHS muestra el comportamiento de la humedad del suelo en una región, el cual se obtiene dividiendo la evapotranspiración real entre la evapotranspiración potencial, cantidades que se estiman de la contabilidad de entradas y salidas, denominado balance hídrico $(1,24,30)$. El IHS calculado para esta localidad indicó que en los meses de diciembre a febrero del primer período, abril y junio del segundo, y entre agosto de 2001 y en febrero de 2002, se

Tabla 1. Características climáticas y de suelos de la Subestación Experimental. Paraguaicito.

\begin{tabular}{lr} 
Localización geográfica & \\
\hline Latitud Norte & $04^{\circ} 23^{\prime}$ \\
Longitud Oeste & $75^{\circ} 44^{\prime}$ \\
Altitud (m) & 1.250
\end{tabular}

Características de suelos

\begin{tabular}{llll}
$\mathrm{pH}$ & 5,7 & Ecotopo & $211 \mathrm{~A}$ \\
Materia Orgánica (\%) & 6,7 & Unidad Cartográfica & Consociación Montenegro \\
Nitrógeno (\%) & 0,28 & Grupo taxonómico & Typic Hapludands \\
Fósforo $(\mathrm{ppm})$ & 2,5 & Material Parental & Cenizas Volcánicas \\
Potasio $\left(\mathrm{cmol}_{(+)} \cdot \mathrm{kg}^{-1}\right)$ & 0,78 & & \\
Calcio $\left(\mathrm{cmol}_{(+)} \cdot \mathrm{kg}^{-1}\right)$ & 2,90 & & \\
${\text { Magnesio }\left(\mathrm{cmol}_{(+)} \cdot \mathrm{kg}^{-1}\right)}^{1,08}$ & & \\
\hline
\end{tabular}



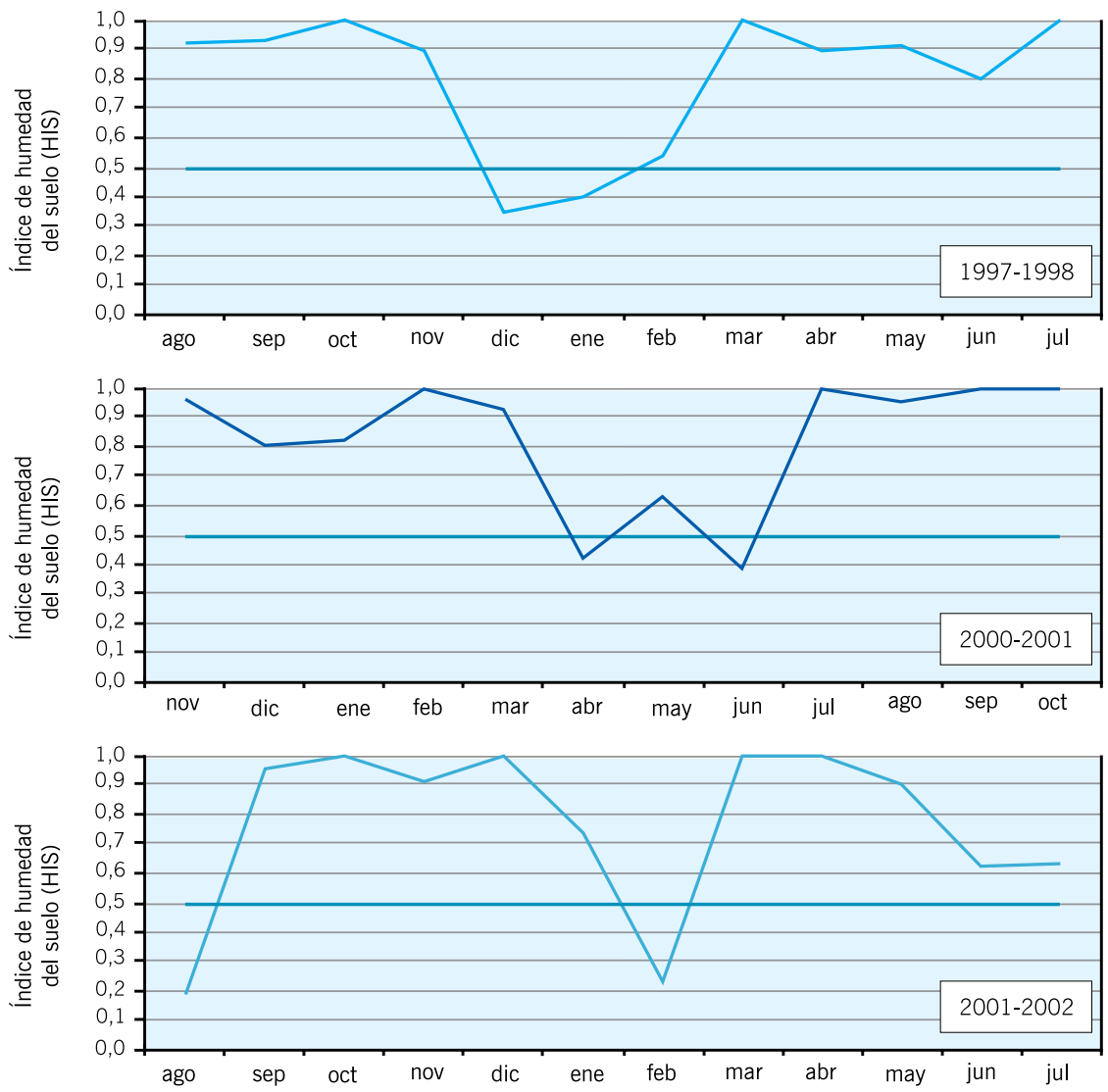

Figura 1. Índice de humedad del suelo (HIS) para tres períodos de estudio (1997/98; 2000/01 y 2001/02), en la Subestación Experimental Paraguaicito. Registros por debajo de la línea azul indican deficiencias hídricas en el suelo.

presentaron deficiencias hídricas en el suelo. Los valores de las variables climáticas para el cálculo del IHS, fueron las registradas en la estación meteorológica de la Subestación Experimental.

Material vegetal. Se emplearon las especies forestales Cordia alliodora (nogal cafetero), Pinus oocarpa (pino) y Eucalyptus grandis (eucalipto) como sombrío del café, y Coffea arabica. c.v. Colombia.

Tratamientos y diseño experimental. Los tratamientos consistieron en el establecimiento de (I) café con sombrío de nogal, (II) café con sombrío de pino, (III) café con sombrío de eucalipto y (IV) café a libre exposición solar. Se empleó un diseño de bloques completos al azar, con cuatro repeticiones, para un total de 16 unidades experimentales. 
Cada unidad experimental contó con 36 árboles de sombrío plantados a 6,0 x 6,0 m, y al interior de este arreglo espacial se estableció el café a 1,5 x 1,5 m (4.500 plantas/ha). Entre bloques se dejaron franjas de 6,0 m de ancho, para reducir el efecto de la sombra proyectada por el sombrío de los bloques contiguos. En términos de área, cada parcela ocupó $1.296 \mathrm{~m}^{2}$.

Los bloques se ubicaron de manera que se maximizaran entre ellos las diferencias topográficas y de uso previo del suelo, mientras se minimizaban las diferencias entre las parcelas que los constituyeron.

Establecimiento y plan de fertilización. El estudio se inició en 1994 con el establecimiento de las especies forestales y un año después (octubre de 1995), se estableció el café. Dos semanas después del establecimiento se realizaron inventarios de supervivencia y se repusieron las plantas faltantes. La distancia de siembra del café fue de 1,5 x 1,5 m (4.500 plantas/ha) y el sombrío a 6,0 x 6,0 m (278 plantas/ha).

Al momento de establecer las especies de sombra se aplicaron 70 g de NPK en formulación 15-38-10, al fondo del hoyo, cabe anotar que no se realizaron posteriores fertilizaciones. Tres meses después de establecido el café se realizó una aplicación de 20 g/planta de Urea, seis meses después se realizó una segunda aplicación del mismo fertilizante, en dosis de $30 \mathrm{~g} /$ planta. El plan anual de fertilización fue el recomendado en el análisis de suelos: $700 \mathrm{~kg}$.ha-1.año-1 de un fertilizante completo, 17-6-18-2; esta dosis se fraccionó en dos aplicaciones en el año, dirigiendo la aplicación al sitio de siembra de la planta.

\section{Variables evaluadas}

Aporte de hojarasca. Para evaluar la cantidad de materia orgánica depositada como hojarasca por las diferentes especies de sombra, se instalaron en cada una de las parcelas experimentales con café y sombrío, cinco trampas de follaje de 0,5 $\mathrm{m}^{2}$ de superficie, cada una. Éstas se instalaron en la primera semana de julio de 1997, dos años y nueve meses después del establecimiento de las especies sombrío, y un año después que Cordia alliodora y Eucalyptus grandis iniciaran el aporte periódico de hojarasca. Entre agosto de 1997 y julio de 1998, se recolectaron mensualmente todas las hojas encontradas en las trampas de follaje.

Con el propósito de observar la evolución de los aportes de hojarasca como función de la edad de las especies, se realizó un segundo muestreo, siete años después de iniciado el estudio (entre noviembre de 2000 y octubre de 2001).

Determinación de la concentración y contenido de nutrientes. Del material vegetal obtenido mensualmente de las trampas de recolección se tomaron tres submuestras de cada especie de sombrío, para determinar la concentración de nutrientes, como son: $\mathrm{N}$ por el método semimicro Kjeldahl, P por colorimetría (Molibdovanadato de amonio), K, Ca y Mg, mediante espectrofotometría de absorción atómica (EAA). También se determinó el contenido de materia orgánica. Las concentraciones de N, P, K, Ca, Mg y cenizas se expresaron en porcentaje (\%). Para determinar el contenido de nutrientes se multiplicó la concentración de éste por el aporte de biomasa seca, en cada tratamiento. Los resultados presentados serán solo de N, P, K, Ca, Mg y materia orgánica, expresados en Mg.ha-1. 
Descomposición del material vegetal residual. El método empleado para la determinación de la tasa de descomposición fue el de "bolsas de descomposición", de acuerdo con Lupwayi y Haque (39). Las bolsas de descomposición se elaboraron con malla plástica de polietileno de $30 \mathrm{~cm} \times 20 \mathrm{~cm}$, con aberturas de malla de 1,0 x 1,0 mm. Se construyeron 504 bolsas, 108 para las especies forestales ( 3 especies forestales $\times 3$ repeticiones $\times 12$ meses) y 144 para el café proveniente de cada sistema de cultivo (4 sistemas de cultivo x 3 repeticiones x 12 meses); también se confeccionaron 252 bolsas, con las mismas características, para reemplazos ante eventuales pérdidas.

El material vegetal (hojas más pecíolos frescos), para el análisis de la tasa de descomposición de la materia seca producida por el café, bajo cada sistema de cultivo, y los producidos por $C$. alliodora, $P$. oocarpa y E. grandis, se tomaron directamente de las plantas de café y de los árboles. El material vegetal no se recolectó del suelo, debido a que no se conocía el momento de su caída y el inicio de su descomposición; el material recolectado se llevó al laboratorio y se secó en una estufa a $80^{\circ} \mathrm{C}$, durante 72 horas. Posteriormente, en cada bolsa se depositaron $100 \mathrm{~g}$ de material vegetal seco de cada una de las especies forestales y el café; y finalmente, las bolsas se ubicaron de manera aleatoria en cada parcela correspondiente.

Durante un período de 365 días (iniciando en agosto de 2001 y finalizando en julio de 2002), cada 30 días, de las parcelas experimentales se recolectaron 21 bolsas (tres por cada especie forestal y tres por cada sistema de cultivo de café). Las muestras se llevaron al laboratorio y se secaron en una estufa a $80^{\circ} \mathrm{C}$, hasta obtener un valor de peso seco constante.

La descomposición del material evaluado mediante la pérdida de peso, correspondiente a cada tiempo de degradación, generó un porcentaje de peso seco remanente (\%Psr), calculado mediante el cociente del peso seco al horno del material remanente mensual $(\boldsymbol{P s m})$, sobre el peso seco al horno del material inicial (Psi):

$$
\% P s r=(P s m / P s i) \times 100
$$

Los $\boldsymbol{P s \boldsymbol { r }}$ se determinaron para calcular la tasa de mineralización o descomposición del material vegetal. Debido a que la descomposición es proporcional al contenido de materia orgánica, la tasa relativa de descomposición o de transferencia de material vegetal mensual hacia el suelo puede ser representada por un modelo exponencial simple $(50,56,63,72$, 73), del orden:

$$
y=y_{0} e^{-k t}
$$

El modelo exponencial simple asume que

$\boldsymbol{y}$ : porcentaje de peso seco remanente o residual

$\boldsymbol{y}_{0}$ : porcentaje de peso seco inicial

$\boldsymbol{t}$ : tiempo

$\boldsymbol{k}$ : tasa relativa de descomposición mensual o constante de velocidad de descomposición del residuo orgánico $(49,56)$. 
El coeficiente de descomposición es un indicador de la "velocidad" con que se transforman o descomponen los residuos vegetales; la tasa de descomposición puede fluctuar entre 0,1/día para residuos de muy rápida descomposición y 0,00001/día en residuos de muy lenta degradación (11).

Coeficiente Isohúmico $\left(\boldsymbol{k}_{1}\right)$. Definido como la constante de transformación en humus de la materia seca aportada o también como la fracción de la materia orgánica que queda sin descomponerse o se descompone muy lentamente después de un período de tiempo dado. Depende esencialmente, pero no exclusivamente, de las características del residuo orgánico $(27,34)$, y debido a la naturaleza de sus componentes, como ligninas y proteínas, Henin y Dupuis (27) y Saña (56), sugieren determinar este coeficiente a través de la fracción orgánica del residuo vegetal, que es relativamente resistente a la descomposición biológica, aplicando la siguiente expresión:

$$
k_{1}=M o_{\text {humificada }} / M o_{\text {aportada }}
$$

Biomasa seca descompuesta e incorporada al suelo. Al final del período de las evaluaciones (12 meses), basados en los aportes totales de hojarasca y otros residuos producidos por las especies forestales y por el café, en sus diferentes sistemas de cultivo, se estimó la cantidad total de biomasa seca descompuesta e incorporada al suelo.

Concentración de nutrientes en la biomasa seca inicial y remanente. Del material vegetal obtenido directamente de los árboles y del café, se tomaron submuestras para determinar la concentración de nutrientes, las cuales se denominaron iniciales (Ci) o en el día cero (0). Con las tres muestras mensuales de cada una de las especies forestales y del café, en cada sistema de cultivo, se conformó una muestra compuesta para su análisis químico. En todos los casos se determinó $\mathrm{N}$ por el método semimicro Kjeldahl, $\mathrm{P}$ por colorimetría (Molibdovanadato de amonio), K, Ca y Mg, mediante espectrofotometría de absorción atómica (EAA), y se determinó contenido de materia orgánica. Las concentraciones de N, P, K, Ca, Mg y cenizas se expresaron en porcentaje (\%).

Liberación de nutrientes ( $\mathbf{N}, \mathbf{P}, \mathbf{K}, \mathbf{C a}$ y $\mathbf{M g}$ ). Para la evaluación de la liberación o transferencia de nutrientes contenidos en la materia seca remanente de todas las especies forestales y del café, en sus diferentes sistemas de cultivo, también se empleó el modelo exponencial simple propuesto por Olson (50), Saña (56), Sinha et al. (63) y Wieder y Lang (72):

$$
W_{f}=W_{i} e^{-k t}
$$

Donde:

$\boldsymbol{w}_{\boldsymbol{f}}$ : cantidad remanente de N, P, K, Ca y Mg

$\boldsymbol{w}_{i}$ : cantidad inicial de cada elemento mineral

$\boldsymbol{k}$ : constante de liberación de nutrientes

$\boldsymbol{t}$ : tiempo de descomposición de la hojarasca expresado en meses.

Análisis de la información. Se realizaron análisis de varianza para todas las variables evaluadas. La comparación de los coeficientes de descomposición de los residuos vegetales 
de las especies forestales y el café, de los coeficientes de liberación de nutrientes y los coeficientes isohúmicos, entre otros, se realizaron mediante pruebas Tukey (5\%). Para evaluar la influencia de la humedad del suelo (IHS) sobre la descomposición de los residuos vegetales, producidos por las especies forestales y el café, en un año, se calcularon los coeficientes de correlación de Pearson.

\section{RESULTADOS Y DISCUSIÓN}

Dinámica en el aporte de hojarasca del café y el componente arbóreo. El aporte de biomasa seca en los sistemas agroforestales se presenta en la Tabla 2, y la dinámica del aporte se presenta en las Figuras 2 y 3 . El promedio del aporte de materia seca (dos recolecciones) en café a libre exposición solar fue de 4,94 t.ha-1 y bajo sombrío de $C$. alliodora, P. oocarpa y E. grandis de 3,79; 4,56 y 4,11 t.ha ${ }^{-1}$, respectivamente. El promedio de la producción de residuos vegetales de nogal, pino y eucalipto fue de 3,46; 6,67 y 6,39 t.ha' ${ }^{-1}$.

En Picea abies, Slodicak et al. (64), reportan producciones entre 8.500 a 8.700 kg.ha-1 de materia seca; Snowdon y Benson (65) indican que la biomasa seca obtenida en Pinus radiata fue de 5,0 t.ha $^{-1}$, y Lugo et al. (38) obtuvieron producciones de biomasa seca de 27,8; 20,4; 10,1; 7,7 y 5,5 t.ha ${ }^{-1}$.año ${ }^{-1}$ en Casuarina equisetifolia, Albizia procera, Eucalyptus robusta, y dos variedades de Leucaena leucocephala, respectivamente, a los cinco años de edad. En sistemas agrosilviculturales donde el componente arbóreo fue Grewia optiva, Celtis australis, Bauhinia variegata, Albizzia chinensis, Toona ciliata y Morus serrata, Parkash et al. (51), estimaron que la producción de biomasa seca fue de 48,0 t.ha $^{-1}$. Puri et al. (53), indican que dependiendo de la densidad de siembra (278, 625 y 2.500 plantas/ha), la producción de biomasa seca producida por Populus deltoides puede variar entre 71,5 y 251,0 t.ha $^{-1}$.

Tabla 2. Aporte de biomasa seca de tres especies forestales y el café, en dos períodos de evaluación. Subestación Experimental Paraguaicito.

\begin{tabular}{lccc} 
& \multicolumn{3}{c}{ Producción de biomasa seca $\left(\mathbf{t}^{\circ}\right.$.ha ${ }^{-1}$ ) } \\
\cline { 2 - 4 } Especies & $1^{\text {er }}$ año & $2^{\circ}$ año & Media \\
Café a libre exposición & $1,47 \mathrm{a}$ & $8,40 \mathrm{a}$ & 4,94 \\
Café + C. alliodora & $1,42 \mathrm{a}$ & $6,16 \mathrm{~b}$ & 3,79 \\
Café + P. oocarpa & $1,61 \mathrm{a}$ & $7,52 \mathrm{ab}$ & 4,56 \\
Café + E. grandis & $1,32 \mathrm{a}$ & $6,90 \mathrm{~b}$ & 4,11 \\
Cordia alliodora & $1,99 \mathrm{a}$ & $4,93 \mathrm{~b}$ & 3,46 \\
Pinus oocarpa* & - & $6,67 \mathrm{ab}$ & 6,67 \\
Eucalyptus grandis & $4,67 \mathrm{a}$ & $8,12 \mathrm{a}$ & 6,40 \\
\hline
\end{tabular}

*De pino se presenta la producción de biomasa seca solo del segundo año. Valores con diferente letra indican diferencias estadísticas. 


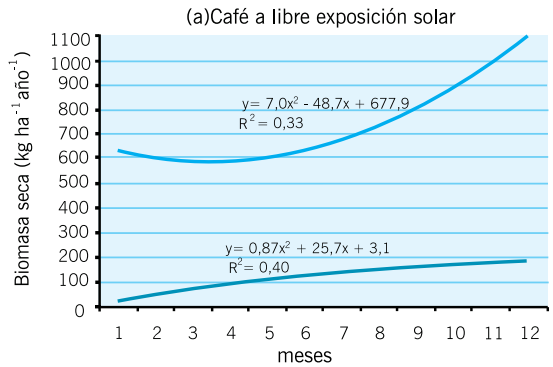

(c) Café con sombrío pino

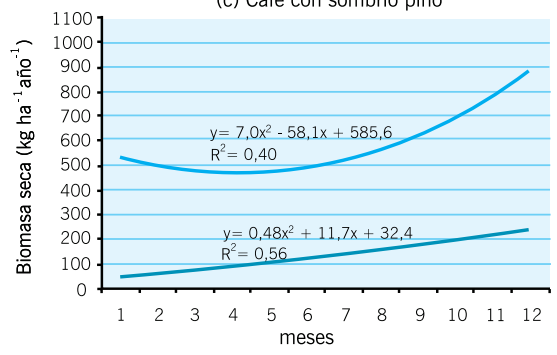

— ago-97 ajul-98

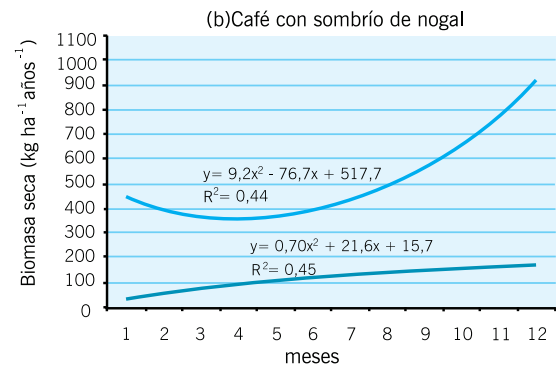

(d) Café con sombrío eucalipto

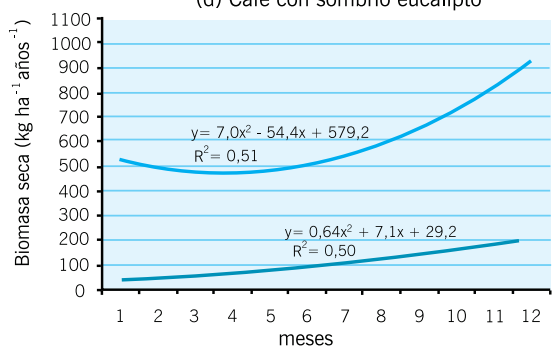

nov-00 a oct-01

Figura 2. Dinámica del aporte de hojarasca por el café cultivado a libre exposición solar y bajo tres especies de sombra y en dos épocas de evaluación, en la Subestación Experimental Paraguaicito.

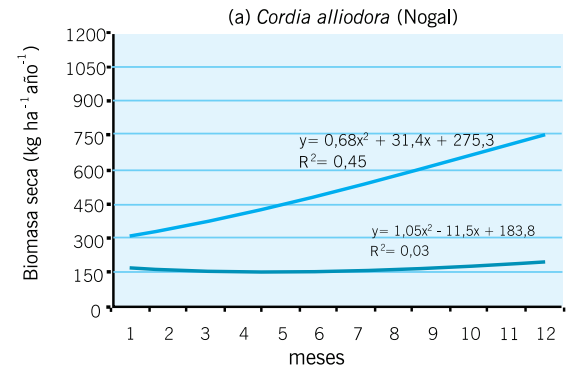

(c) Eucalyptus grandis (Eucalipto)

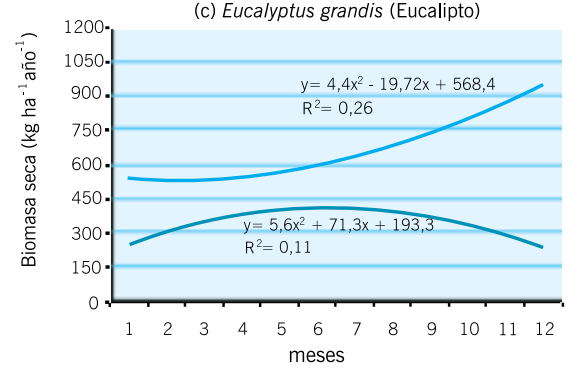

(b) Pinus oocarpa (Pino)

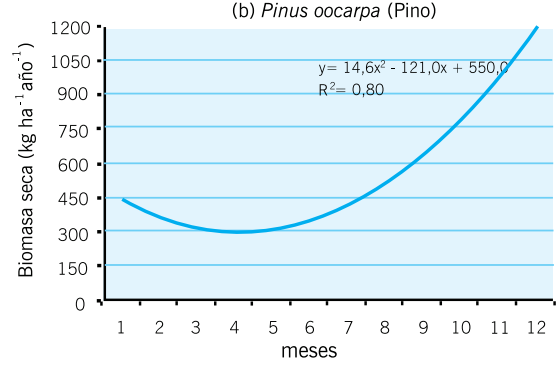

— ago-97 a jul-98 — nov-00 a oct-01

Figura 3. Dinámica del aporte de hojarasca por las especies forestales empleadas como sombrío del café y en dos épocas de evaluación, en la Subestación Experimental Paraguaicito. 
La dinámica en el aporte de hojarasca por el café en cada sistema de cultivo, independiente de si se siembra a libre exposición solar o bajo sombrío, indica que en la primera recolección de hojarasca (agosto de 1997 y julio de 1998), las mayores producciones de residuos vegetales se presentaron en febrero y entre junio y julio de 1998, las deficiencias de agua en el suelo para esta fase de las evaluaciones, se registraron en los meses de diciembre a febrero. En el segundo período de evaluación (noviembre de 2000 y octubre de 2001), los mayores aporte de biomasa seca por el café se presentaron entre mayo y agosto de 2001, y las tasas más bajas de estos aportes se registraron entre noviembre y diciembre del mismo año, con deficiencias hídricas registradas entre abril y junio.

El nogal cafetero, empleado como sombrío del café bajo las condiciones de la S. E. Paraguaicito, tuvo sus mayores aporte de biomasa seca durante los meses mayo a julio (55,2 $\mathrm{kg}$ ha $\left.^{-1}\right)$ de su primera evaluación, mientras que el lapso de menor producción de residuos fue entre febrero y abril $\left(459,8 \mathrm{~kg}^{\mathrm{h}} \mathrm{ha}^{-1}\right)$. En la segunda evaluación la mayor producción de hojarasca se registró en los mismos meses $\left(2.056,3 \mathrm{~kg}^{-h^{-1}}\right)$ y los meses de menor aporte fueron agosto a octubre $\left(969,8 \mathrm{~kg} \mathrm{ha}^{-1}\right)$.

El pino, en su único período de evaluaciones, hizo sus mejores aportes de hojarasca en el lapso entre mayo y julio $\left(2.986,0 \mathrm{~kg}^{-\mathrm{ha}^{-1}}\right)$, con un descenso en su transferencia de material orgánico, en los meses de noviembre de 2000 a enero de 2002 (928,0 kg.ha-1). En cuanto al comportamiento productivo de hojarasca por parte del eucalipto, en su primera evaluación, realizó los mayores aportes en los meses de diciembre de 2007 y febrero de $2008\left(1.247,3 \mathrm{~kg}\right.$ ha $\left.^{-1}\right)$ y los menores entre mayo y julio de 2001 y agosto de 2000 (801,5 kg.ha-1); en el segundo período (2000-2001) la mayor cantidad se registró en los meses de mayo a julio $\left(2.660,5 \mathrm{~kg} \cdot \mathrm{ha}^{-1}\right)$ y la más baja producción en los meses de noviembre a diciembre de 2000 y enero de 2001 (1.648,5 kg.ha-1 $)$.

El que se hallan registrado diferencias de un mes entre la ocurrencia de picos en el aporte de hojarasca entre el eucalipto y el nogal, como consecuencia de la disminución en la precipitación, pone de manifiesto dos cosas: (I) la mayor sensibilidad de Eucalyptus grandis a períodos de déficit hídrico, indicado por la rápida respuesta en el aporte de hojarasca, a pesar de que la humedad del suelo es mayor en las parcelas de eucalipto que en las de nogal, y (II) la existencia de un mecanismo de control que le permite a esta especie reducir las pérdidas de agua por transpiración (70).

La existencia de tal mecanismo ha sido comprobada en otras especies del género Eucalyptus en diversos estudios realizados en India, Israel y Australia, países donde no obstante las limitaciones en pluviosidad (menos de $700 \mathrm{~mm}$ de lluvia al año), especies como Eucalyptus tereticornis y Eucalyptus camaldulensis se usan a gran escala en la recuperación de dunas y en el establecimiento de bosques dendroenergéticos (21).

Peso seco residual (Psr) de las muestras de las tres especies forestales y el café. El peso seco medio de las muestras remanentes de café a libre exposición, a los 30, 180 y 365 días, fue de 93,0; 60,5 y 34,5 g, respectivamiente; para café con sombrío de nogal fue de 89,7; 51,0 y 33,6 g, en café bajo sombrío de pino de 93,0; 49,0 y 36,8 g, y en café con sombrío de eucalipto los valores fueron de 94,$0 ; 62,0$ y $30,1 \mathrm{~g}$, respectivamente (Figura 4). 

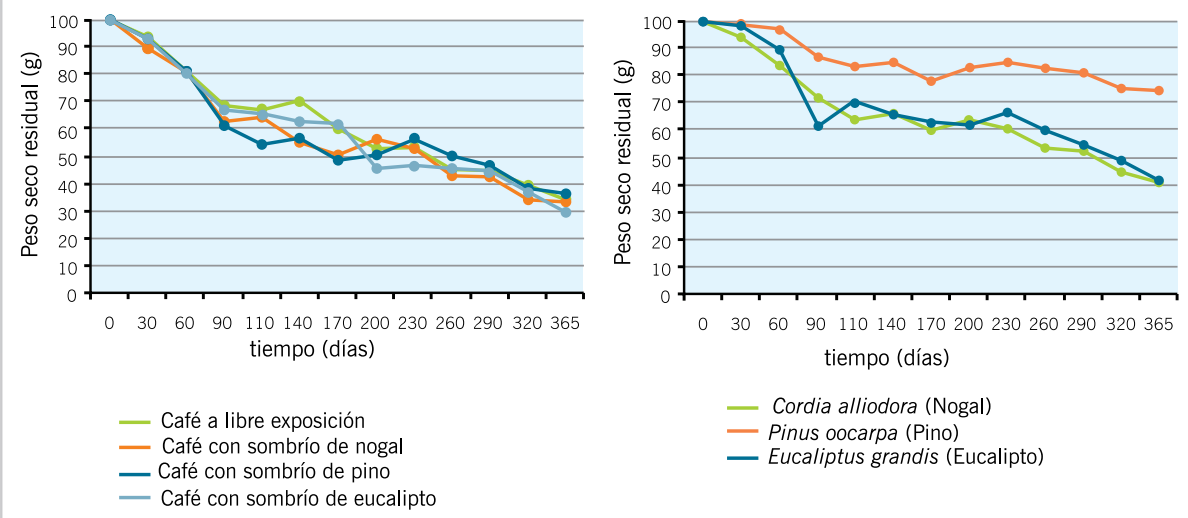

Figura 4. Peso seco residual (g) de las muestras vegetales de café a libre exposición y bajo sombrío y de las tres especies forestales en descomposición, durante un período de un año.

En estos períodos de tiempo las muestras remanentes de nogal fueron de 94,$3 ; 60,5$ y $41,5 \mathrm{~g}$, respectivamente; las de pino de 98,$7 ; 78,5$ y 74,6 g, y las de eucalipto de 98,3 ; 63,0 y 42,1 g. El peso seco de la muestra inicial fue de $100 \mathrm{~g}$ en todas las especies. En descomposición de residuos de Pinus pinaster, P. nigra, Adenocarpus decorticans y Cistus laurifolius, Moro y Domingo (45) registraron pesos secos de 80 y $90 \mathrm{~g}$ en las muestras remanentes a los 180 y 360 días en las especies de pino, y entre 50 y 80 g a los 180 días y 25 y $60 \mathrm{~g}$ a los 360 días, en las otras dos especies.

Lusk et al. (40) reportan valores de pesos secos remanentes de $60 \mathrm{~g}$ en $P$. radiata, en un período de cinco meses, y Callahan et al. (6), en Pinus sp. obtuvieron pesos remanentes de 85 y 75 g a los 200 y 300 días de descomposición de los residuos. White et al. (71) y Sariyildiz (57), reportan pesos remanentes en la muestras de Pinus sp. de $55 \mathrm{~g}$ a los 360 días y de 75 y $60 \mathrm{~g}$ a los 180 y 360 días de descomposición de $P$. sylvestris. Singh et al. (62), después de 365 días de descomposición de residuos de Azdirachta indica, Dalbergia sissoo, Pongamia pinnata y Shorea robusta, obtuvieron porcentajes de masa remanentes de $27,2 \%, 31,9 \%, 41,2 \%$ y $52,9 \%$, respectivamente.

Descomposición de la hojarasca. En la Tabla 3 y la Figura 5, se presenta de acuerdo al modelo aplicado (exponencial simple), la tasa relativa de descomposición (k) para el café a libre exposición solar y bajo sombrío, así como de nogal, pino y eucalipto. Modelos similares fueron aplicados por Heuveldop et al. (29) en C. alliodora, por Gama-Rodrigues et al. (22) en Peltogyne angustiflora, Centrolobium robustum, Arapatiella psilophylla, Sclerolobium chrysophyllum, C. trichotoma y Macrolobium latifolium, por Prause y Lifschitz (52) en Gleditsia amorphoides, Patagonula americana, Chlorophora tinctoria y Astronium balansae, y por Munguía et al. (46) en Eucalyptus deg/upta, Erythrina poeppigiana y café, entre otros. 
Tabla 3. Valores estimados de la tasa de descomposición mensual del follaje de café a libre exposición solar y bajo sombrío y de $C$. alliodora, $P$. oocarpa y E. grandis.

\begin{tabular}{lcc}
\multicolumn{1}{c}{ Tratamientos } & Tasa de descomposición de residuos \\
\cline { 2 - 3 } Café a libre exposición & K.año-1 & C.v. \\
Café con sombrío de nogal & $1,00 \mathrm{a}$ & 0,04 \\
Café con sombrío de pino & $1,01 \mathrm{a}$ & 0,37 \\
Café con sombrío de eucalipto & $0,87 \mathrm{a}$ & 0,12 \\
C. alliodora (nogal) & $1,08 \mathrm{a}$ & 0,22 \\
P. oocarpa (pino) & $0,78 \mathrm{a}$ & 0,11 \\
E. grandis (eucalipto) & $0,26 \mathrm{~b}$ & 0,03 \\
\hline
\end{tabular}

k: Tasa de descomposición anual; c.v. Coeficiente de variación

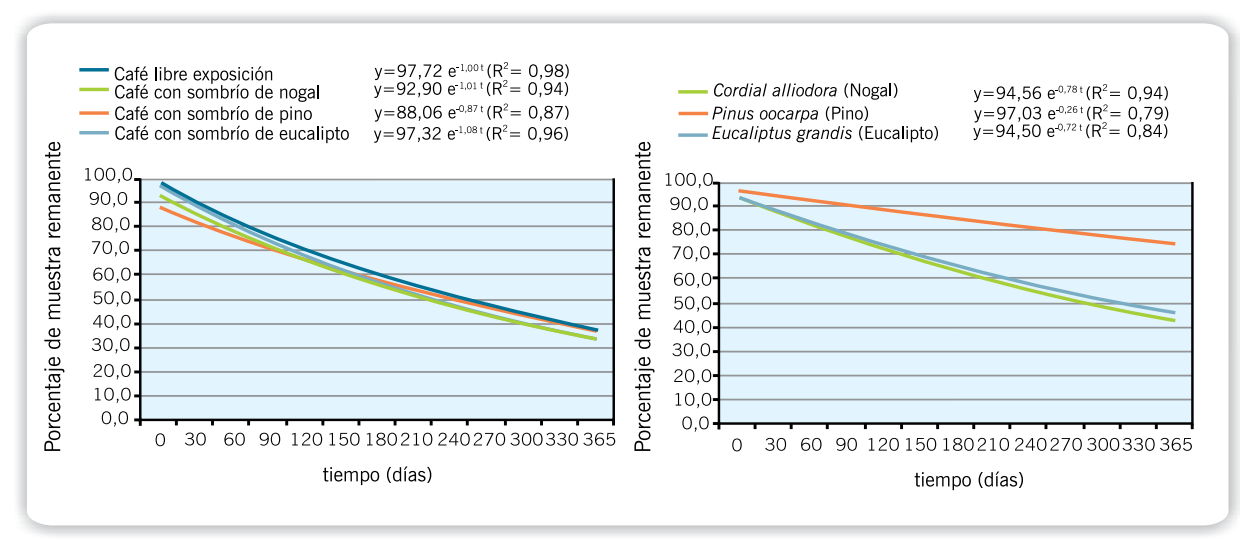

Figura 5. Porcentaje de masa remanente del follaje de café a libre exposición solar y bajo sombrío de especies forestales, de Cordia alliodora, Pinus oocarpa y Eucalyptus grandis.

El análisis de varianza y a las pruebas de comparación (Tukey al 5\%), no mostraron diferencias estadísticas entre la tasa de descomposición anual para el café a libre exposición solar y bajo sombrío; tampoco fue evidente esta diferencia en la rata de descomposición de los residuos de nogal y eucalipto. Hubo diferencia estadística entre la tasa de descomposición de los residuos vegetales de pino y el café bajo todos sus ambientes, y nogal y eucalipto, es decir, que los residuos vegetales de pino se descomponen mas lentamente que el resto de los materiales.

El promedio de la tasa de descomposición $(k$. año-1) de los residuos vegetales del café a libre exposición y bajo sombrío fue de 0,99; la de nogal de 1,08 y la de pino y eucalipto de 0,26 y 0,72 respectivamente. La tasa de descomposición reportada por Ribeiro et al. 
(54), en E. g/obulus oscila entre 0,37 y 0,42 ; y en E. Globulus, Guo y Sims (25) encontraron tasas de descomposición medias de 0,01 y 1,34. En otras especies como Metrosideros polymorpha, Austin y Vitousek (3), obtuvieron que la tasa de descomposición (k) fluctúo entre 0,22 y 1,06. Moro y Domingo (45) registraron valores de $k$ de 0,13 y 0,16 en P. pinaster y P. Nigra, y en C. trichotona, Gamma-Rodrigres (22) fijó un $k$ de 1,2. Al ajustar modelos exponenciales en la tasa de descomposición de 51 especies arbóreas, Simmons y Hawkins (61) encontraron que la $k$. año-1 fluctuaba entre 0,002 y 0,45. Esta variación en la tasa de descomposición, de acuerdo con Berg (4), y la cual puede decrecer 0,1\% a 0,00001\% por día, puede deberse a la composición química del material vegetal o calidad del sustrato.

Santa (55) reportó índices de descomposición $\left(k \cdot \mathrm{mes}^{-1}\right)$ en $P$. pinea y $P$. pinaster de -0,15 y -0,08 y Kwabiah etal. (33), aplicando modelos exponenciales simples, en descomposición de residuos de Croton megalocarpus, Sesbania sesban y Calliandra calothyrsus, obtuvieron coeficientes de descomposición de 0,037, 0,042 y 0,012, respectivamente.

Coeficiente Isohúmico $\left(\boldsymbol{k}_{\mathbf{1}}\right)$. Las pruebas de comparación realizadas (Tukey $5 \%$ ) entre los coeficientes Isohúmicos $\left(\boldsymbol{k}_{\mathbf{1}}\right)$ de café a libre exposición solar y bajo sombrío, no evidenciaron diferencia estadística, lo que indica que la fracción no descompuesta del material vegetal del café no es determinada por el tipo de sombrío; el $\boldsymbol{k}_{1}$ en un período de un año de evaluación fue de 0,37 para el café a libre exposición solar y bajo sombrío (Tabla 4).

La fracción no descompuesta para C. alliodora fue del $46 \%$, la cual no fue estadísticamente diferente de la estimada en $E$. grandis (49\%), estas dos presentaron diferencia estadística (prueba Tukey 5\%) al compararse con la de P. oocarpa (77\%). Ribeiro et al. (54) observaron que $E$. globulus después de 645 días de descomposición, conservaba el $52,8 \%$ del material inicial; Fasbender (17) determinó que la fracción no descompuesta en residuos de C. alliodora, en un período de 300 días, fue del 50,8\%, y Arguello (2) indica que al finalizar la semana 12 de descomposición de residuos de especies como Erythrina sp., ésta conservaba más del $61 \%$ del peso inicial sin descomponer.

Tabla 4. Coeficiente isohúmico de los residuos de café a libre exposición solar, café bajo sombrío y de las tres especies forestales.

\begin{tabular}{lcc}
\multicolumn{1}{c}{ Tratamientos } & Coeficiente Isohúmico $\left(\boldsymbol{k}_{\mathbf{1}}\right)$ & c.v. \\
\hline Café a libre exposición & $0,37 \mathbf{a}$ & 0,13 \\
Café con sombrío de nogal & $0,36 \mathbf{a}$ & 0,42 \\
Café con sombrío de pino & $0,42 \mathbf{a}$ & 0,14 \\
Café con sombrío de eucalipto & $0,34 \mathbf{a}$ & 0,17 \\
Cordia alliodora (nogal) & $0,46 \mathbf{a}$ & 0,14 \\
Pinus oocarpa (pino) & $0,77 \mathbf{~ b}$ & 0,01 \\
Eucalyptus grandis (eucalipto) & $0,49 \mathbf{a}$ & 0,16 \\
\hline
\end{tabular}

c.v.: Coeficiente de variación 
Tasa de incorporación de residuos vegetales. En la Figura 6, se presentan los porcentajes de transferencia o incorporación mensual al suelo de residuos vegetales descompuestos, basados en los modelos ajustados y de acuerdo con las condiciones climáticas de la localidad.

En los primeros 30 días de descomposición del material vegetal aportado por el café a libre exposición solar $(4,94$ t.ha-1.año-1 $)$ y bajo sombrío $\left(4,15\right.$ t.ha- ${ }^{-1}$.año-1) se incorporó al suelo el $9,8 \%$ y el $14,3 \%$. La incorporación del $50 \%$ de los residuos vegetales se registró a los 240 días de descomposición y a los 365 días se degradó e incorporó al suelo el $64,1 \%$ y el $65,4 \%$ de la materia orgánica producida por el café a libre exposición solar y bajo sombrío, respectivamente.

Del material vegetal aportado por el nogal $\left(3,46\right.$ t.ha $^{-1}$.año $\left.{ }^{-1}\right)$, el pino $\left(6,67\right.$ t.ha ${ }^{-1}$.año-1 $)$ y el eucalipto $\left(6,39\right.$ t.ha $^{-1}$.año- $)$, en los primeros 30 días de descomposición se incorporó al suelo el $11,2 \%$, el $5,0 \%$ y el 10,8\%, respectivamente. La incorporación del $50 \%$ de los residuos vegetales de nogal y el $51 \%$ de los residuos de eucalipto se registró entre los 300 y 330 días de descomposición, y a los 365 días se degradó e incorporó al suelo el $56,7 \%$, el $25,2 \%$ y el $54,0 \%$ de la materia orgánica producida por nogal, pino y eucalipto, respectivamente.

Munguía et al. (46), obtuvieron tasas de descomposición del 23\% a los 213 días en E. deg/upta y del 60\% en 213 días en Coffea arabica. En P. radiata, Ganjegunte et al. (23) obtuvieron ratas de descomposición del follaje del $24 \%$ anual, y le atribuyeron a compuestos polifenólicos y a las grandes concentraciones de lignina, la baja tasa de descomposición. Santa (55), en Quercus rotundifolia, P. pinea y P. pinaster, registró que cerca del $30 \%$ de la materia seca se descompone durante los primeros 4 meses. Hartemink y O'Sullivan (26) en Piper aduncum, Gliricidia sepium e Imperata cylindrica encontraron que la tasa de descomposición fue del $50 \%$ en las diez primeras semanas. Ribeiro et al. (54) obtuvieron que la descomposición de E. globulus fue del 47,2\% en 645 días. Moro
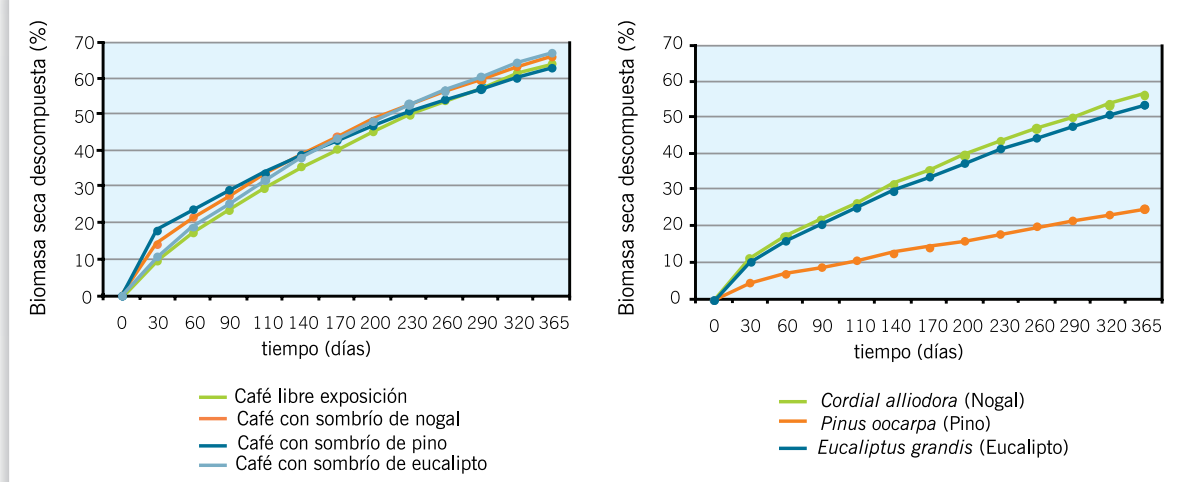

Figura 6. Porcentajes de la biomasa inicial descompuesta e incorporada al suelo mensualmente. 
y Domingo (45) registraron porcentajes de descomposición del 14,0\% en 360 días en $P$. pinaster y P. Nigra, y el tiempo de descomposición total puede fijarse en 4 a 5 años; y Gama-Rodrigues et al. (22), encontraron que en un año se descompuso e incorporó al suelo el $60 \%$ de los residuos de C. tricotoma.

Prause y Lifschitz (52), afirman que las hojas de las distintas especies forestales se descomponen a diferentes velocidades en un proceso rápido al comienzo, con registros de pérdidas de peso entre $28 \%$ y $60 \%$ del peso total del material vegetal, en los primeros tres meses. En un bosque compuesto por Acacia polyphylla, Alchornea triplinervia, Aspidosperma polyneuron, Bastardiopsis densiflora, Cordia trichotoma, Gallesia integrifolia, Luehea divaricata y Machaerium brasiliense, entre otros, Tundis et al. (69) encontraron que el coeficiente de descomposición $(k)$ fue de 1,71 y que el tiempo necesario para la descomposición era del 50\% y 95\%, después de 150 y 639 días, respectivamente.

Descomposición de residuos vs. Índice de humedad del suelo (IHS). La influencia de la humedad del suelo sobre la proporción de la biomasa seca descompuesta mensualmente, entre agosto de 2001 y julio de 2002, se presenta en la Tabla 5.

El coeficiente de correlación ( $r$ ) entre el IHS y el porcentaje de la materia seca que se descompone cada mes en café a libre exposición solar fue de -0,18, en café bajo sombrío de 0,05, en nogal y pino de 0,31 y en eucalipto de 0,21, pero los análisis de varianza no indicaron relación alguna entre estas dos variables ( $>0,05$ en todos los casos), lo que indica que la disponibilidad o no de agua en el suelo no tuvo influencia sobre la descomposición de la masa vegetal, en las condiciones ambientales y de suelos donde se desarrolló el estudio. No obstante, algunos autores sostienen que el clima modifica notablemente la rapidez de la descomposición de los residuos vegetales en la superficie del suelo, ejerciendo influencia sobre el tipo y abundancia de la materia orgánica $(5,32)$. La humedad y la temperatura se encuentran entre las variables más determinantes,

Tabla 5. Relación entre la humedad del suelo sobre el porcentaje mensual de biomasa seca descompuesta, entre agosto de 2001 y julio de 2002.

\begin{tabular}{|c|c|c|c|c|c|c|c|c|c|c|c|c|c|}
\hline \multirow{3}{*}{ Especies } & \multicolumn{12}{|c|}{ Índice de Humedad del Suelo (IHS) } & \multirow{3}{*}{$r$} \\
\hline & Ago & Sep & Oct & Nov & Dic & Ene & Feb & Mar & Abr & May & Jun & Jul & \\
\hline & $0,20 *$ & 0,96 & 1,00 & 0,92 & 1,00 & 0,74 & 0,24 & 1,00 & 1,00 & 0,91 & 0,63 & 0,64 & \\
\hline \multicolumn{14}{|c|}{ Porcentaje de la biomasa descompuesta mensualmente } \\
\hline $\begin{array}{l}\text { Café al } \\
\text { sol }\end{array}$ & 6,7 & 11,8 & 12,9 & 1,5 & $-3,2$ & 9,8 & 7,5 & 0,0 & 7,9 & 0,5 & 5,0 & 5,2 & $-0,18$ \\
\hline $\begin{array}{l}\text { Café/ } \\
\text { sombra }\end{array}$ & 7,9 & 11,6 & 16,6 & 2,3 & 3,5 & 4,1 & 2,8 & $-1,0$ & 5,5 & 1,8 & 8,2 & 3,2 & 0,05 \\
\hline Nogal & 5,7 & 10,4 & 12,0 & 7,8 & $-2,4$ & 6,0 & $-3,5$ & 3,0 & 7,1 & 1,2 & 7,9 & 3,4 & 0,31 \\
\hline Pino & 1,3 & 1,5 & 10,2 & 3,2 & $-1,1$ & 6,3 & $-5,0$ & $-1,5$ & 2,3 & 1,3 & 6,0 & 1,0 & 0,31 \\
\hline Eucalipto & 1,8 & 8,6 & 27,6 & $-8,2$ & 4,2 & 3,1 & 0,5 & $-4,5$ & 6,5 & 5,6 & 5,6 & 7,3 & 0,21 \\
\hline
\end{tabular}


porque influyen en el desarrollo de la vegetación y en la actividad de los microorganismos.

Bunvong y Granger (5) citan que la intensidad máxima de la descomposición de la materia orgánica se observa en condiciones de temperatura moderada (alrededor de $30^{\circ} \mathrm{C}$ ) y con un contenido de humedad del suelo alrededor del 60-80\% de su capacidad máxima de retención de agua. En otros estudios McTiernan (42) en P. sylvestris, y Liski et al. (36) en Pinus sp., observaron que la tasa de descomposición de los residuos vegetales presentaba una correlación positiva con la humedad relativa, la evapotranspiración, la precipitación y la temperatura. Guo y Sims (25), observaron que la tasa de descomposición de residuos vegetales de $E$. globulus, presentaba una correlación positiva con factores como la precipitación, la temperatura y la radiación solar.

Camiré et al. (7) y Simmons y Hawkins (61), en la evaluación de 51 especies arbóreas, encontraron correlaciones positivas entre la tasa de descomposición y los factores climáticos, temperatura y precipitación; y Austin y Vitousek (3) observaron que la precipitación tiene una correlación positiva con la tasa de descomposición de residuos vegetales de Metrosideros polymorpha. Dora et al. (15), en Peltogyne gracilipes, obtuvieron relaciones inversas entre la tasa de descomposición y la distribución de lluvias. Thaiutsa y Granger (68) aplicaron regresiones lineales simples para relacionar la descomposición de la hojarasca en bosques tropicales y variables climáticas, de éstas obtuvieron que los porcentajes de descomposición aumentan con la precipitación y la humedad relativa, y que la temperatura no tenía influencia sobre la tasa de descomposición.

Pero la tasa de descomposición no depende solo de los factores ambientales como la temperatura, la humedad y la precipitación y de las características del suelo, de su humedad y de las poblaciones microbianas $(13,16)$; las diferencias en descomposición de los materiales vegetales están en función de la composición química, de las concentraciones de los nutrientes y de la relación C:N $(31,48)$. Sariyildiz (57) indica que la concentración de $\mathrm{N}$ y la relación $\mathrm{C}: \mathrm{N}$ se han identificado como los factores más importantes que controlan los procesos de descomposición de la materia orgánica.

Relación Carbono:Nitrógeno (C:N). La relación C: $\mathrm{N}$ depende de las especies y la edad de las mismas, y es un buen indicador de la susceptibilidad de la hojarasca a ser degradada. El rango óptimo en los residuos orgánicos se encuentra entre 25-30:1; si el residuo de partida es rico en $\mathrm{C}$ y pobre en $\mathrm{N}$, la descomposición será lenta, pero por el contrario, con altas concentraciones de $\mathrm{N}$, éste se transformará en amoníaco, impidiendo la correcta actividad biológica. Si el material final obtenido tras la descomposición tiene un valor C:N alto ( $>35$ ), indica que no ha sufrido una descomposición completa y si el índice es muy bajo $(<25)$, puede ser por una excesiva mineralización, aunque todo ello depende de las características del material inicial $(13,17,48)$. En la Tabla 6 , se presentan las concentraciones iniciales y finales de $\mathrm{C}$ orgánico y $\mathrm{N}$, y la relación $\mathrm{C}: \mathrm{N}$ del café a libre exposición solar y bajo sombrío, y de las tres especies forestales.

La relación C: $\mathrm{N}$ inicial en el café a libre exposición solar fue de 32,2 y la final de 19,8 a los 365 días de descomposición del material vegetal. Estas relaciones de café bajo sombrío fueron de 24,9 y 16,9 respectivamente. Los coeficientes de correlación entre el tiempo de descomposición de la biomasa seca del café en los dos sistemas de cultivo (libre exposición 
Tabla 6. Concentración inicial (Ci) y final (Cf) de carbono orgánico (CO), nitrógeno (N) y la relación $\mathrm{C}: \mathrm{N}$ del material remanente del café a libre exposición solar, café con sombrío, y de las especies forestales nogal, pino y eucalipto.

\begin{tabular}{lccc|ccc|cccc}
\hline \multirow{2}{*}{ Especies } & \multicolumn{3}{c|}{ CO (\%) } & \multicolumn{3}{c|}{ N (\%) } & \multicolumn{3}{c}{ C:N } \\
\cline { 2 - 11 } & Ci & Cf & r & Ci & Cf & r & Inicial & Final & r \\
\hline Café solo & 53,4 & 49,5 & $-0,49$ & 1,7 & 2,5 & 0,68 & 32,2 & 19,8 & $-0,69$ \\
Café con sombrío & 53,3 & 50,1 & $-0,85$ & 2,1 & 3,0 & 0,75 & 24,9 & 16,9 & $-0,80$ \\
C. alliodora & 47,1 & 42,5 & $-0,75$ & 2,0 & 2,4 & 0,06 & 23,8 & 18,1 & $-0,43$ \\
P. oocarpa & 55,7 & 55,4 & $-0,38$ & 0,5 & 0,8 & 0,48 & 105,1 & 73,9 & $-0,38$ \\
E. grandis & 55,2 & 52,6 & $-0,88$ & 0,8 & 1,4 & 0,91 & 71,7 & 37,3 & $-0,90$ \\
\hline
\end{tabular}

solar y bajo sombra), fueron de -0,69 en el primer caso y de -0,80 en el segundo, indicando una relación inversa entre estas dos variables, es decir, que la relación C:N disminuye al incrementarse el tiempo de descomposición de los residuos del café.

Las relaciones $\mathrm{C}: \mathrm{N}$ iniciales en los residuos de $C$. alliodora, $P$. oocarpa y $E$. grandis fueron de 23,8; 105, 1 y 71,7 y las relaciones C: $\mathrm{N}$ finales (a los 365 días de descomposición de la biomasa seca) de 18,$1 ; 76,9$ y 37,3 respectivamente. Los coeficientes de correlación entre el tiempo de descomposición de la materia seca fueron de -0,43 en nogal, de -0,38 en pino y de -0,90 en eucalipto, indicando una relación inversa entre estas dos variables, es decir, que la relación C: $\mathrm{N}$ disminuye al incrementarse el tiempo de descomposición de los residuos vegetales. La alta relación C: $\mathrm{N}$, estimada para el $P$. oocarpa, indica una lenta descomposición de sus residuos vegetales. Gama-Rodrigues et al. (22) reportan relaciones C:N de 27,3 para C. tricotoma, y Sariyildiz (57) indican relaciones de 5,3 para P. sy/vestris; sin embargo, White et al. (71) encontraron en $P$. rigida, relaciones iniciales C:N de 95,0 y finales (863 días) de 0,39.

$\mathrm{XU}$ et al. (73) obtuvieron correlaciones positivas entre la masa remanente y la relaciones C:N y C:P, después de dos años de descomposición de residuos de Castanopsis sieboldii y Schima wallichii; Moro y Domingo (45) y Corbeels (11), afirman que la descomposición de residuos vegetales de $P$. pinaster y $P$. nigra, después de dos años, correlaciona positivamente con la relación C: $\mathrm{N}$ inicial. Por lo general, el índice de descomposición es más alto en las especies que tienen un máximo de ceniza y de nitrógeno, y una mínima relación C: $\mathrm{N}$ y de lignina (5).

Concentraciones iniciales y finales de nutrientes. Las concentraciones iniciales ( 0 días) y las finales (365 días) de N, P, K, Ca y Mg, en las muestras remanentes de café a libre exposición y bajo sombrío, y de las tres especies forestales, se presentan en la Tabla 7.

Coffea arabica. En café a libre exposición solar las concentraciones iniciales de N, P, K, Ca y $\mathrm{Mg}$ fueron de 2,82\%, 0,19\%, 1,23\% 1,70\% y 0,33\% y las finales de 2,82\%, 0,11\%, 0,14\% 3,0\% y 0,35\%, respectivamente; y los r entre el tiempo de descomposición y las concentraciones de nutrientes fueron de $-0,40 ; 0,20 ; 0,81 ;-0,81$ y $-0,41$, lo que 


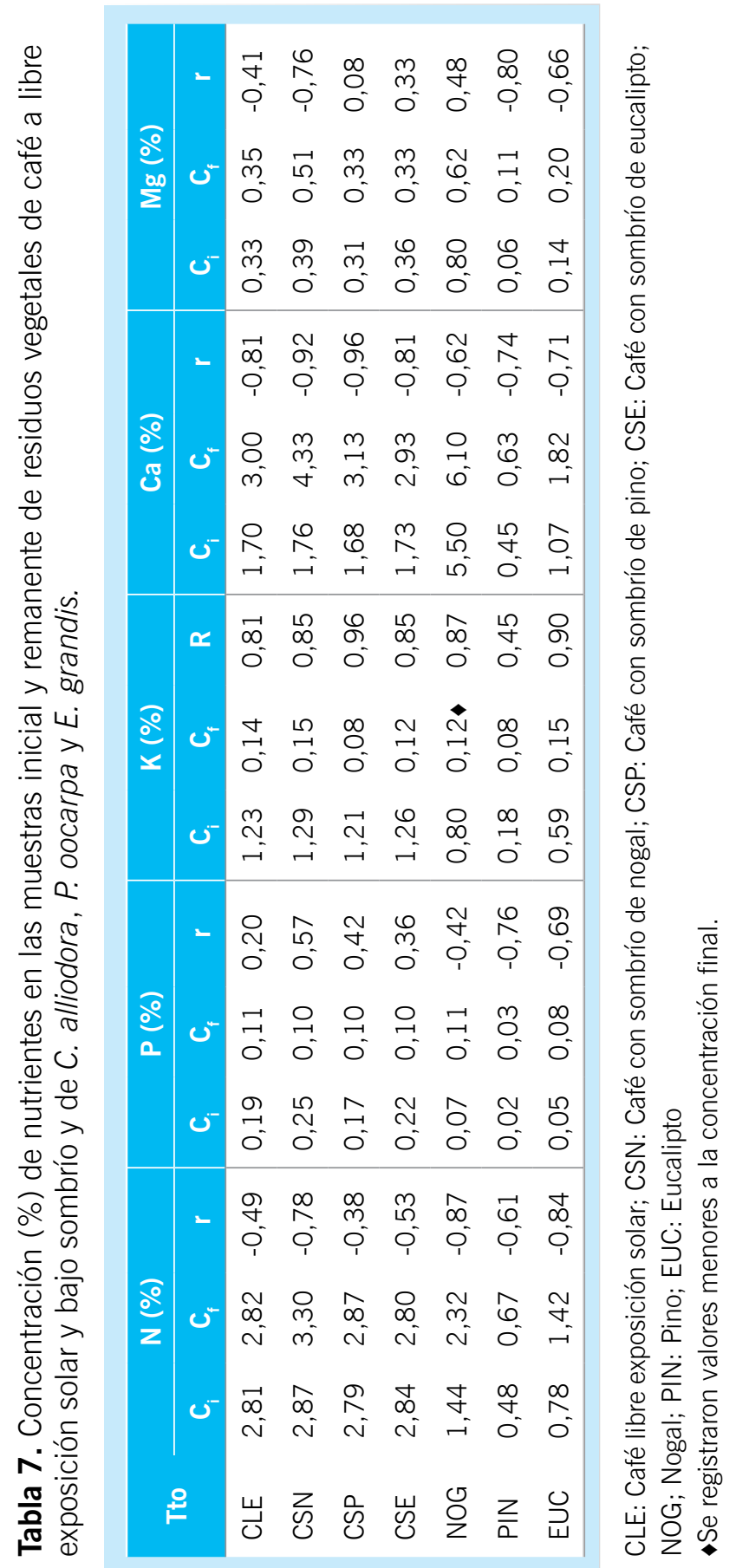


indica que las concentraciones de N, Ca y Mg aumentan y las de P y K disminuyen, al reducirse el porcentaje de material vegetal remanente. Las concentraciones medias iniciales y finales de $\mathrm{N}, \mathrm{P}, \mathrm{K}$, Ca y $\mathrm{Mg}$ del café bajo los tres sistemas de sombrío tuvieron el mismo comportamiento que a libre exposición solar.Las concentraciones iniciales de $\mathrm{N}$, P, K, Ca y Mg fueron de 1,44\%, 0,07\%, 0,8\%, 5,50\% y 0,80\%, respectivamente y las finales de $2,32 \%, 0,11 \%, 0,12 \%, 6,10 \%$ y 0,62\%. Los coeficientes de correlación entre el porcentaje de muestra residual y las concentraciones de nutrientes fueron $-0,87 ;-0,42$; 0,87 ; -0,62 y 0,48, e indican que las concentraciones $N$, P y Ca aumentan al disminuir el porcentaje de material vegetal remanente y las de $\mathrm{K}$ y Mg disminuyen. Gama-Rodrigues et al. (22), obtuvieron relaciones similares entre la concentración de $\mathrm{N}$ y el porcentaje de muestra remanente, en la descomposición del materia vegetal de C. trichotoma.

Pinus oocarpa. Las concentraciones iniciales de N, P, K, Ca y Mg fueron de 0,48\%, $0,02 \%, 0,18 \%, 0,45 \%$ y $0,06 \%$, respectivamente y las finales de 0,67\%; 0,03\%; 0,08\%, 0,63\% y 0,11\%. Los coeficientes de correlación entre el porcentaje de muestra residual y las concentraciones de estos elementos, con valores de $-0,61 ; 0,54 ; 0,45$; $-0,74$ y $-0,80$, indican que las concentraciones $N$, Ca y Mg aumentan al disminuir el porcentaje de material vegetal restante, mientras que las concentraciones de $\mathrm{P}$ y $\mathrm{K}$ disminuyen.

McTiernan (42) en Pinus sylvestris, Santa (39) en $P$. pinea, P. pinaster y P. nigra, Moro y Domingo (45) en $P$. pinaster y P. nigra, White et al. (71) en P. rigida, Zhi-an Li et al. (75) en Acacia mangiun, A. auriculaeformis, Eucalyptus citriodora, P. ellioti y Schima superba, y Montagnini et al. (44) en Stryphnodendron excelsum, Vochysia ferruginea, Vochysia hondurensis y Hyeronima alchorneoides, observaron que las concentraciones de $\mathrm{N}$ presentaban una correlación inversa con el porcentaje de la masa residual, en la descomposición de estos materiales vegetales.

Eucalyptus grandis. En eucalipto las concentraciones iniciales de N, P, K, Ca y Mg fueron de $1,42 \%, 0,08 \%, 0,15 \%, 1,07 \%$ y $0,14 \%$, respectivamente y las finales de $1,42 \%$, $0,08 \%, 0,15 \%, 1,82 \%$ y 0,20\% para los mismos elementos. Los coeficientes de correlación entre el porcentaje de muestra residual y las concentraciones de estos nutrimentos $(-0,84 ;-0,69 ; 0,90 ;-0,71$ y -0,66), indican que las concentraciones $N, P$, Ca y Mg aumentan al disminuir el porcentaje de material vegetal remanente y que las concentraciones de $\mathrm{K}$ disminuyen al reducirse este porcentaje.

Se han encontrado relaciones inversas entre la masa vegetal residual y las concentraciones de $\mathrm{N}$, y relaciones directas con las concentraciones de $\mathrm{P}, \mathrm{K}$ y Ca en la descomposición de residuos de E. globulus y Metrosideros polymorpha (3, 54). Montagnini et al. (44) en la descomposición de residuos de Stryphnodendron excelsum, Vochysia ferruginea, Vochysia hondurensis y Hyeronima alchorneoides, observaron que en las dos especies de Vochysia la concentración de $\mathrm{P}$ aumentó y en Stryphnodendron excelsum y en Hyeronima alchorneoides disminuyó al descomponerse el material vegetal. Ribeiro et al. (54) no obtuvieron correlaciones entre la muestra residual mensual y la concentración de Mg en descomposición de residuos de Eucalyptus globulus, y Dora et al. (15), en Peltogyne gracilipes, obtuvieron correlaciones positivas entre la tasa de descomposición y las concentraciones de Mg. 
Liberación de nutrientes. La dinámica en la transferencia de nutrientes ( $\boldsymbol{k}$ ), a los 365 días de descomposición de la hojarasca del café a libre exposición y bajo sombrío, y de las tres especies forestales, se presenta en la Tabla 8 y la Figura 7.

Nitrógeno. La transferencia de $\mathrm{N}$ del café bajo sombrío de eucalipto fue mayor que la de café bajo sombrío de nogal; la liberación de $\mathrm{N}$ por los residuos de café a libre exposición solar fue igual a la tasa de transferencia del café bajo las diferentes especies de sombrío. La dinámica en la transferencia de $\mathrm{N}$ fue igual en $C$. alliodora y $E$. grandis y hubo inmovilización del $\mathrm{N}$ en $P$. oocarpa. La hojarasca de café transfiere $\mathrm{N}$ a una tasa más alta que la transferida por la hojarasca de las especies forestales. Munguía (46), obtuvo tasas de liberación ( $k$. día ${ }^{-1}$ ) de $N$ en $E$. deg/upta y C. arabica de 0,06 y 0,034.

Fósforo. No hubo diferencias estadísticas en cuanto a la liberación de $\mathrm{P}$ por la hojarasca del café en descomposición, en los cuatro sistemas de cultivo. C. alliodora transfiere P a una tasa más alta que P. oocarpa, mientras que se presenta inmovilización de este nutriente en $E$. grandis. Las tasas de liberación de $P$ fueron mayores en café a libre exposición solar y bajo sombrío de eucalipto, que las tasas de transferencia de la hojarasca de $C$. alliodora y $P$. oocarpa. Munguía (46), obtuvo tasas de liberación ( $k$.día $\left.{ }^{-1}\right)$ de $P$ en $E$. deg/upta y C. arabica de 0,12 y -0,035.

Tabla 8. Tasas de transferencia de nutrientes $(k$. año-1 $)$, a los 365 días de descomposición de la hojarasca del café a libre exposición solar, café bajo sombrío, y de las tres especies forestales.

\begin{tabular}{|c|c|c|c|c|c|c|}
\hline \multirow{2}{*}{ Especies } & \multicolumn{5}{|c|}{ Tasa de liberación de nutrientes $(k$. año-1) } & \multirow{2}{*}{$p$} \\
\hline & $\mathbf{N}$ & $\mathbf{P}$ & $\mathbf{K}$ & $\mathrm{Ca}$ & Mg & \\
\hline $\begin{array}{l}\text { Café a libre } \\
\text { exposición }\end{array}$ & $-0,061 a b$ & $-0,082 a b$ & $-0,248 a b$ & $-0,020$ bc & $-0,054$ bcd & * \\
\hline $\begin{array}{l}\text { Café con sombrío } \\
\text { de nogal }\end{array}$ & $-0,056 \mathbf{b}$ & $-0,122 \mathbf{a}$ & $-0,233 a b$ & $-0,003 d$ & $-0,049 \mathbf{c d}$ & * \\
\hline $\begin{array}{l}\text { Café con sombrío } \\
\text { de pino }\end{array}$ & $-0,064 a b$ & $-0,084 a b$ & $-0,320$ a & $-0,022 \mathbf{b}$ & $-0,087 \mathrm{abc}$ & * \\
\hline $\begin{array}{l}\text { Café con sombrío } \\
\text { de eucalipto }\end{array}$ & $-0,073 \mathbf{a}$ & $-0,106 \mathbf{a}$ & $-0,257 a b$ & $-0,045 \mathbf{a}$ & $-0,111 \mathbf{a}$ & * \\
\hline $\begin{array}{l}\text { Cordia alliodora } \\
\text { (Nogal) }\end{array}$ & $-0,015 \mathbf{c}$ & $-0,050 \mathbf{b}$ & $-0,225 a b$ & $-0,039 \mathbf{a}$ & $-0,091 \mathbf{a b}$ & * \\
\hline $\begin{array}{l}\text { Pinus oocarpa } \\
\text { (Pino) } \\
\text { Eucaliptus }\end{array}$ & $0,006 \mathbf{d}+$ & $-0,002 \mathbf{c}$ & $-0,086 \mathbf{c}$ & $-0,003 \mathbf{d}$ & $0,014 \mathbf{e}$ & * \\
\hline $\begin{array}{l}\text { grandis } \\
\text { (Eucalipto) }\end{array}$ & $-0,003 \mathbf{c d}$ & $0,011 \mathrm{c}$ & $-0,170$ bc & $-0,006 \mathbf{c d}$ & $-0,026 \mathbf{d}$ & * \\
\hline
\end{tabular}

+ Valores positivos indican inmovilización del nutriente

- Valores de $k$ seguidos por letra distinta en la misma columna indican diferencia estadística según prueba Tukey $5 \%$

$*<0,001$ en todos los casos 


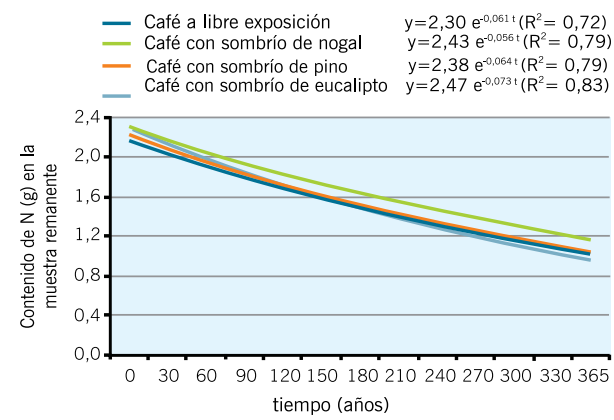

- Café a libre exposición - Café con sombrío de nogal

- Café con sombrío de pino

$y=0,11 e^{-0,082 t}\left(R^{2}=0,57\right)$ $y=0,14 e^{-0,122 t}\left(R^{2}=0,76\right)$ $y=0,11 e^{-0,084 t}\left(R^{2}=0,66\right)$

Café con sombrío de eucalipto $y=0,134 \mathrm{e}^{-0,106 t}\left(R^{2}=0,72\right)$

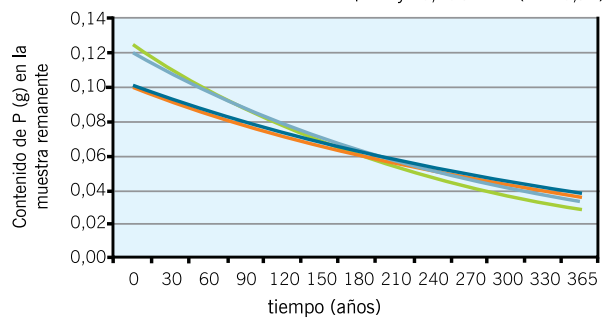

- Café a libre exposición

- Café con sombrío de noga

- Café con sombrío de pino

$y=0,90 e^{-0,248 t}\left(R^{2}=0,75\right)$ $\mathrm{y}=0,68 \mathrm{e}^{-0,233 t}\left(R^{2}=0,71\right)$ $y=1,00 e^{-0,320 t}\left(R^{2}=0,80\right)$

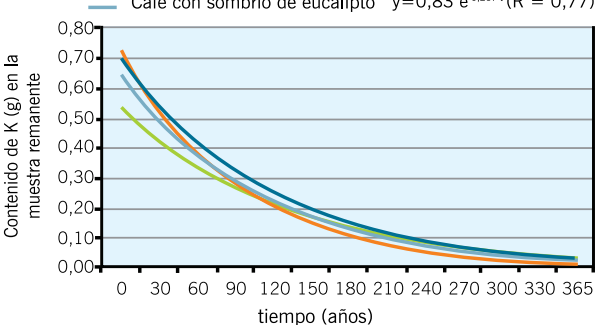

- Cordial alliodora (Nogal)

- Pinus oocarpa (Pino)

$y=1,31 e^{-0,015 t}\left(R^{2}=0,23\right)$

$y=0,50 e^{-0,006 t}\left(R^{2}=0,02\right)$

- Eucaliptus grandis (Eucalipto) $\mathrm{y}=0,77 \mathrm{e}^{-0,003 t}\left(\mathrm{R}^{2}=0,01\right)$
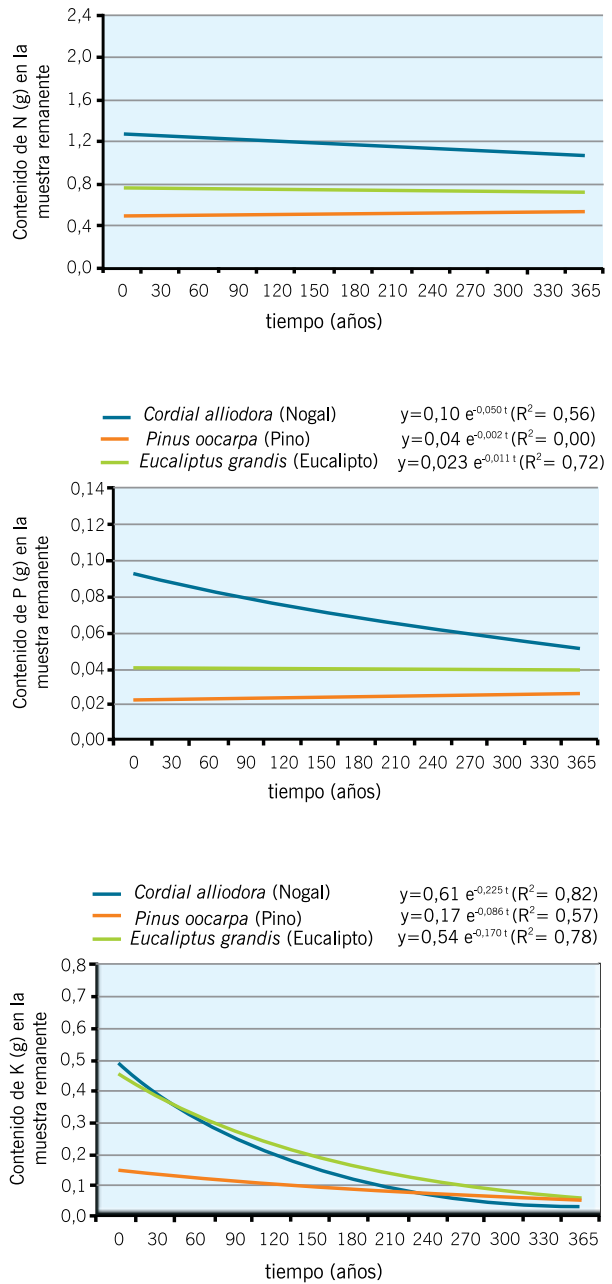

Continúa... 

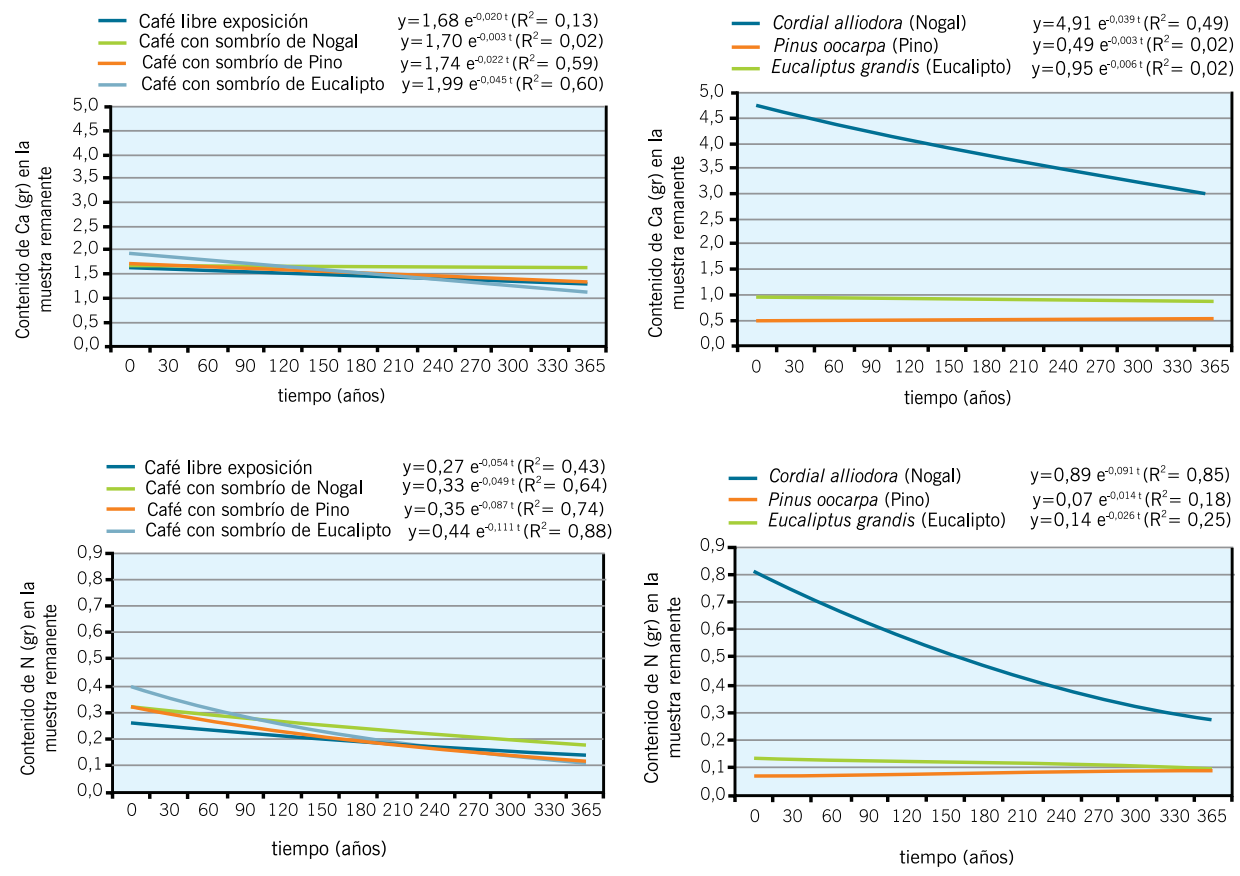

Figura 7. Dinámica en la transferencia de N, P, K, Ca y Mg ( $k$.año-1) por el café, en cuatro sistemas de cultivo, y por las tres especies forestales, en la Subestación Experimental Paraguaicito.

Potasio. No hubo inmovilización de $\mathrm{K}$ en café bajo sus diferentes sistemas de cultivo, ni en las especies forestales. Las tasas de liberación de $\mathrm{K}$ fueron iguales en la hojarasca del café (bajo sombra y a libre exposición) al compararlas con las de $C$. alliodora. Las tasas de liberación de $\mathrm{K}$ de la hojarasca producida por $P$. oocarpa y $E$. grandis fueron iguales, y presentaron diferencias con respecto a las de café y nogal. Munguía (46), obtuvo tasas de liberación ( $k$.día $\left.{ }^{-1}\right)$ de $\mathrm{K}$ en $E$. deg/upta y $C$. arabica de $-0,49$ y -0,56, respectivamente.

Calcio. Las mayores tasas de liberación de Ca se presentaron en la hojarasca de C. alliodora y en la de café bajo sombrío de eucalipto; no obstante, a pesar de que el café y las especies forestales no presentaron inmovilización de $\mathrm{Ca}$, las tasas más bajas de liberación de este nutriente se registraron en la hojarasca de café bajo sombrío de nogal, en pino y eucalipto. 
Magnesio. Los residuos de café bajo sombrío de eucalipto presentaron una tasa de liberación de Mg más alta que la hojarasca del café bajo sombrío de nogal y a libre exposición solar. Entre las especies forestales la tasa de transferencia más baja fue en $E$. grandis al compararse con $C$. alliodora, mientras que en $P$. oocarpa se presentó inmovilización de este nutrimento.

De este estudio pudo establecerse que

- La producción media de biomasa seca en café fue de 4,35 t.ha-1, en C. alliodora de 3,46 t.ha-1 , en P. oocarpa de 6,67 t.ha-1 y en E. grandis de 6,39 t.ha-1.

- El modelo que mejor representó la tasa de descomposición de los residuos vegetales fue el exponencial simple.

- La tasa relativa de descomposición mensual $(\boldsymbol{k})$ en café a libre exposición solar fue de 1,0; en café bajo sombrío fluctúo entre 0,87 y 1,08; en $C$. alliodora fue de 0,78; en $P$. oocarpa de 0,26 y en E. grandis de 0,72.

- El sistema de cultivo de café, libre exposición solar o bajo sombrío, no afecta la tasa de descomposición de sus residuos vegetales.

- Los residuos vegetales de $P$. oocarpa se descomponen más lentamente $(k=0,20)$ que los residuos de $C$. alliodora y $E$. grandis.

- En un período de 365 días se descompone e incorpora al suelo el 65,1\% de los residuos producidos por café, el 56,7\% de los producidos por C. alliodora, el 25,2\% de los producidos por $P$. oocarpa y el $54,0 \%$ de los producidos por $E$. grandis.

- La fracción de la materia orgánica no descompuesta $\left(\boldsymbol{k}_{\mathbf{1}}\right)$ en un período de 365 días fluctuó entre el $34 \%$ y el $42 \%$ en café bajo sus diferentes sistemas de cultivo; en $C$. alliodora fue del $46 \%$, en P. oocarpa del $77 \%$ y en E. grandis del $49 \%$.

- La disponibilidad o no de agua en el suelo no tiene influencia sobre la descomposición de la masa vegetal en estos sistemas agroforestales.

- En un período de 365 días de descomposición, la biomasa seca producida por el café transfiere al suelo el 64,8\% del N, el 82,9\% del P, el 96,7\% del K, el 34,3\% del Ca y el $63,1 \%$ del $\mathrm{Mg}$, contenido en los residuos vegetales.

- En este mismo período, la descomposición de los residuos vegetales producidos por $C$. alliodora, transfiere al suelo el 33,3\% del N, el 54,4\% del P, el 93,6 del K, el 54,0\% del Ca y el 67,7\% del Mg contenido en estos residuos.

- Al finalizar los 365 días, los residuos vegetales producidos por P. oocarpa, no transfirieron al suelo N, P, Ca y Mg, solamente liberaron el 64,5\% del K presente en los residuos.

- En un período de 365 días de descomposición, la biomasa seca producida por E. grandis transfiere al suelo el $23,6 \%$ del $\mathrm{N}$, el 35,5\% del $\mathrm{P}$, el $89,3 \%$ del $\mathrm{K}$, el $28,5 \%$ del Ca y el 39,9\% del Mg contenido en los residuos vegetales. 
- Aporte de hojarasca y contenido de nutrientes de Eucalyptus grandis, Pinus chiapensis, Pinus tecunumanii e Inga densiflora, empleadas como sombrío en café (Coffea arabica).

Descripción del sitio de estudio. El estudio se realizó en la finca La Suecia, ubicada en el municipio de El Tambo, departamento del Cauca, zona cafetera sur de Colombia y bajo la coordinación de la Subestación Experimental El Tambo. Las características geográficas, climáticas $(5,9,11)$ y de suelos $(20)$ se presentan en la Tabla 9.

Índice de Humedad del Suelo (IHS). El Índice de humedad del suelo para el sitio de estudio en El Tambo (Cauca), se presenta en la Figura 8; el IHS es la relación entre la Evapotranspiración real y la Potencial, estimadas a partir de un balance hídrico (30). La región sur de la zona cafetera colombiana presenta una estación seca desde mediados de junio a mediados de septiembre y una estación Iluviosa de octubre a junio. La distribución de la cantidad de lluvia en esta región se registra de la siguiente manera: $45 \%$ en el

Tabla 9. Características climáticas y de suelos de la localidad.

\begin{tabular}{llll}
\multicolumn{2}{c}{ Localización geográfica } & \multicolumn{2}{c}{ Características de suelos } \\
\hline Latitud & $2^{\circ}$ 29' Norte & $\mathrm{pH}$ & 5,0 \\
Longitud & $76^{\circ} 50^{\prime}$ Oeste & Materia Orgánica (\%) & 16,2 \\
Altitud (m) & 1.693 & Nitrógeno (\%) & 0,6 \\
& & Fósforo $(\mathrm{ppm})$ & 1,0 \\
& & Potasio $\left(\mathrm{cmol}_{(+)} \mathrm{kg}^{-1}\right)$ & 0,20 \\
Características climáticas & & Calcio $\left(\mathrm{cmol}_{(+)} \mathrm{kg}^{-1}\right)$ & 0,5 \\
\hline Temperatura $\left({ }^{\circ} \mathrm{C}\right)$ & 19,6 & Magnesio $\left.\left(\mathrm{cmol}_{(+)}\right) \mathrm{kg}^{-1}\right)$ & 0,3 \\
Precipitación (mm) & 1.885 & Ecotopo & $112 \mathrm{~B}$ \\
Brillo Solar (horas año) & 1.545 & Unidad Cartográfica & \\
Humedad Relativa (\%) & 78,2 & Grupo taxonómico & Melanudands \\
& & Material Parental & Cenizas Volcánicas \\
& &
\end{tabular}

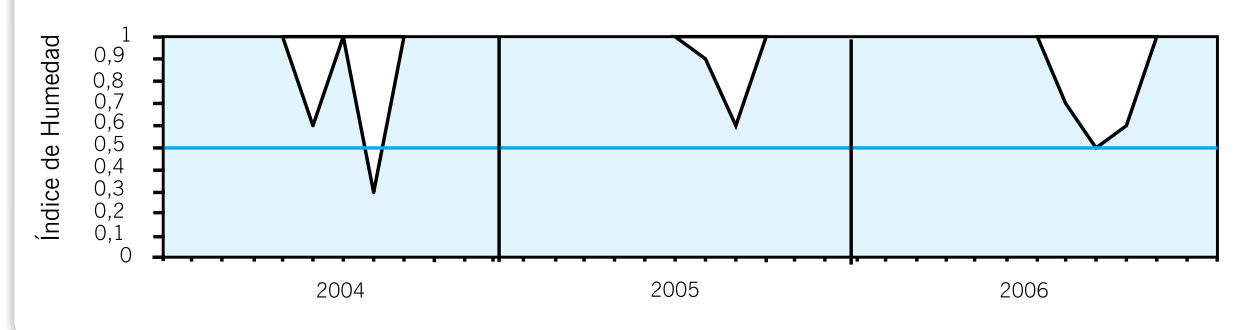

Figura 8. Índice de humedad del suelo (HIS), período 2004 a 2006, en la finca La Suecia (Cauca). Registros por debajo de la línea roja, indican deficiencias hídricas en el suelo. 
primer semestre y 55\% en el segundo del año (30), este patrón de distribución de lluvias se presenta en la zona de ubicación de la finca La Suecia. Los años 2004 y 2006 se caracterizaron por presentar un período seco entre julio y agosto, sin que llegaran a presentarse deficiencias hídricas severas.

Material vegetal. Se emplearon las especies forestales Eucalyptus grandis, Pinus chiapensis, Pinus tecunumanii e Inga densiflora como sombrío del café, Coffea arabica. c.v. Colombia

Tratamientos y diseño experimental. Los tratamientos consistieron en el establecimiento de (I) café con sombrío de E. grandis; (II) Café con sombrío de P. chiapensis; (III) Café con sombrío de P. tecunumanii; (IV) Café con sombrío de I. densiflora y (V) café a libre exposición solar. Se empleó un diseño de bloques completos al azar, con cuatro repeticiones, para un total de 20 unidades experimentales.

Cada unidad experimental contó con 36 árboles de sombrío plantados a 6,0 x 6,0 m; al interior de este arreglo espacial se plantó el café a 1,5 x 1,5 m (4.500 plantas/ha); entre cada bloque se dejaron franjas de 6,0 $\mathrm{m}$ de ancho para reducir el efecto de la sombra proyectada por el sombrío de los bloques contiguos. En términos de área, cada parcela ocupó $1.296 \mathrm{~m}^{2}$. Los bloques se ubicaron de manera que se maximizaran entre ellos las diferencias topográficas y de uso previo del suelo, mientras se minimizaban las diferencias entre las parcelas que los constituyen.

Establecimiento. El estudio se inició en mayo de 1999, con el establecimiento de las especies forestales y la leguminosa, y continuó dos años después con el establecimiento del café, con el objeto de reducir el efecto que sobre el desarrollo inicial del café tendría del crecimiento diferencial en altura, y por ende, en la sombra producida, de las especies de sombrío. Dos semanas después del establecimiento de los árboles, se realizaron inventarios de supervivencia y se repusieron las plántulas faltantes.

Evaluaciones. Para evaluar la cantidad de materia orgánica depositada como hojarasca por las diferentes especies de sombra, se instalaron en cada una de las 16 parcelas experimentales con café y sombrío, cinco trampas de follaje de 0,5 $\mathrm{m}^{2}$ de superficie cada una. Estas trampas se instalaron en la última semana de diciembre de 2003, cuatro años y siete meses después del establecimiento de las especies sombrío. Entre enero de 2004 y diciembre de 2006, se recolectaron mensualmente, todas las hojas encontradas en las trampas de follaje. Las muestras separadas por trampa y especie se secaron al horno, se pesaron y se analizaron para determinar su concentración y contenido de nutrientes.

Determinación de la concentración y contenido de nutrientes. Para la determinación de la concentración y el contenido de nutrientes se procedió de igual forma que en el estudio anterior, realizado en la Subestación Experimental Paraguaicito en el Quindío.

Análisis de la información. Para determinar la significancia estadística de las diferencias entre aportes de hojarasca, concentración y contenido de nutrientes, se realizaron análisis de varianza y pruebas de comparación Tukey (5\%). 


\section{RESULTADOS Y DISCUSIÓN}

Aporte de hojarasca. En la Tabla 10 se presenta el aporte mensual de hojarasca producida por E. grandis, P. chiapensis, P. tecunumanii e I. densiflora.

Primera recolección (2004). E. grandis tuvo sus tasas máximas de aporte de hojarasca en los meses de diciembre y enero, tiempo en el cual aportó el 28,9\% (2.287,5 kg.ha-1) del total de la hojarasca producida en este año; en el mes de octubre no produjo hojarasca, fenómeno que coincidió con la deficiencia hídrica registrada en el mes de agosto (Figura 9). P. chiapensis presentó su mayor producción de biomasa seca entre los meses de agosto y noviembre, lapso en el cual aportó el 49,7\% (782,9 kg. ha- $\left.{ }^{-1}\right)$ de la biomasa seca total producida en el año, las deficiencias hídricas se presentaron en agosto; en los meses de mayo a junio esta especie de pino no produjo hojarasca, sin embargo, este hecho no tuvo relación con deficiencias hídricas.

Entre julio, agosto y noviembre, P. tecunumanii produjo $117,8 \mathrm{~kg} \cdot \mathrm{ha}^{-1}$ de hojarasca, correspondientes al 55,9\% del total producido en al año, esta biomasa se observa después de la deficiencia hídrica calculada en agosto. En el mes de mayo se produjo la menor cantidad biomasa, que fue solo del 2,3\% (48,8 $\left.\mathrm{kg} \mathrm{ha}^{-1}\right)$ del total producido en el año. Al igual que todas las otras especies, I. densiflora presentó su mayor cantidad de hojarasca entre los meses de agosto y noviembre, la cual fue del 27,0\% $\left(875,2 \mathrm{~kg}^{-h^{-1}}\right)$ de la biomasa seca total producida en este año, mientras que la producción más baja se registró en los meses de febrero y octubre, con un promedio de $62,0 \mathrm{~kg} \cdot \mathrm{mes}^{-1}$. No obstante el lapso tan corto que presentó la deficiencia hídrica registrada (agosto), ésta parece haber influido en la pérdida de follaje de las especies arbóreas empleadas como sombrío en café.

Segunda recolección (2005). En este segundo período de evaluaciones no se evidenciaron deficiencias hídricas en la zona de estudio (Figura 9). E. grandis presentó su mayor aporte de hojarasca $(2.439,6 \mathrm{~kg})$ en los meses de febrero y abril, lo que equivale al $24,6 \%$ del total aportado en el año; en el mes de octubre se registró la menor producción de biomasa seca, solo el 3,4\% (340,5 kg.ha-1) del total producido en el año. P. chiapensis produjo el $33,2 \%\left(1.184,1 \mathrm{~kg}^{2} \mathrm{ha}^{-1}\right)$ de biomasa seca en los meses de julio y septiembre y las producciones más bajas de material vegetal se registraron en el mes de abril y entre los meses de enero a marzo, períodos en los cuales sólo produjo el $8,4 \%$ y el $5,5 \%$ del material vegetal seco producido en 2005.

P. tecunumanii produjo su mayor cantidad de biomasa seca $\left(2.752,5 \mathrm{~kg}^{-h^{-1}}\right)$ equivalente al $34,7 \%$ del total, entre los meses de agosto a octubre; mientras que en el mes de mayo $(7,7 \%)$ y entre los meses de enero a marzo $(15,0 \%)$ se registraron las producciones más bajas de hojarasca. En el segundo período de evaluación, I. densiflora presentó su mayor aporte de hojarasca en los meses de julio y septiembre con el 38,0\% (1.984,3 kg.ha-1) de la biomasa total producida en el año. En marzo se produjo la menor cantidad de material vegetal, que fue solo del $4,8 \%$.

Tercera recolección (2006). E. grandis presentó sus tasas máximas de aporte de hojarasca en los meses de enero y marzo, tiempo en el cual aportó el 27,4\% $\left(2.575,8 \mathrm{~kg} \cdot \mathrm{ha}^{-1}\right)$ del total de la hojarasca producida. En octubre sólo produjo el 0,3\% de hojarasca $\left(25,6 \mathrm{~kg}\right.$. ha-1 ${ }^{-1}$, 


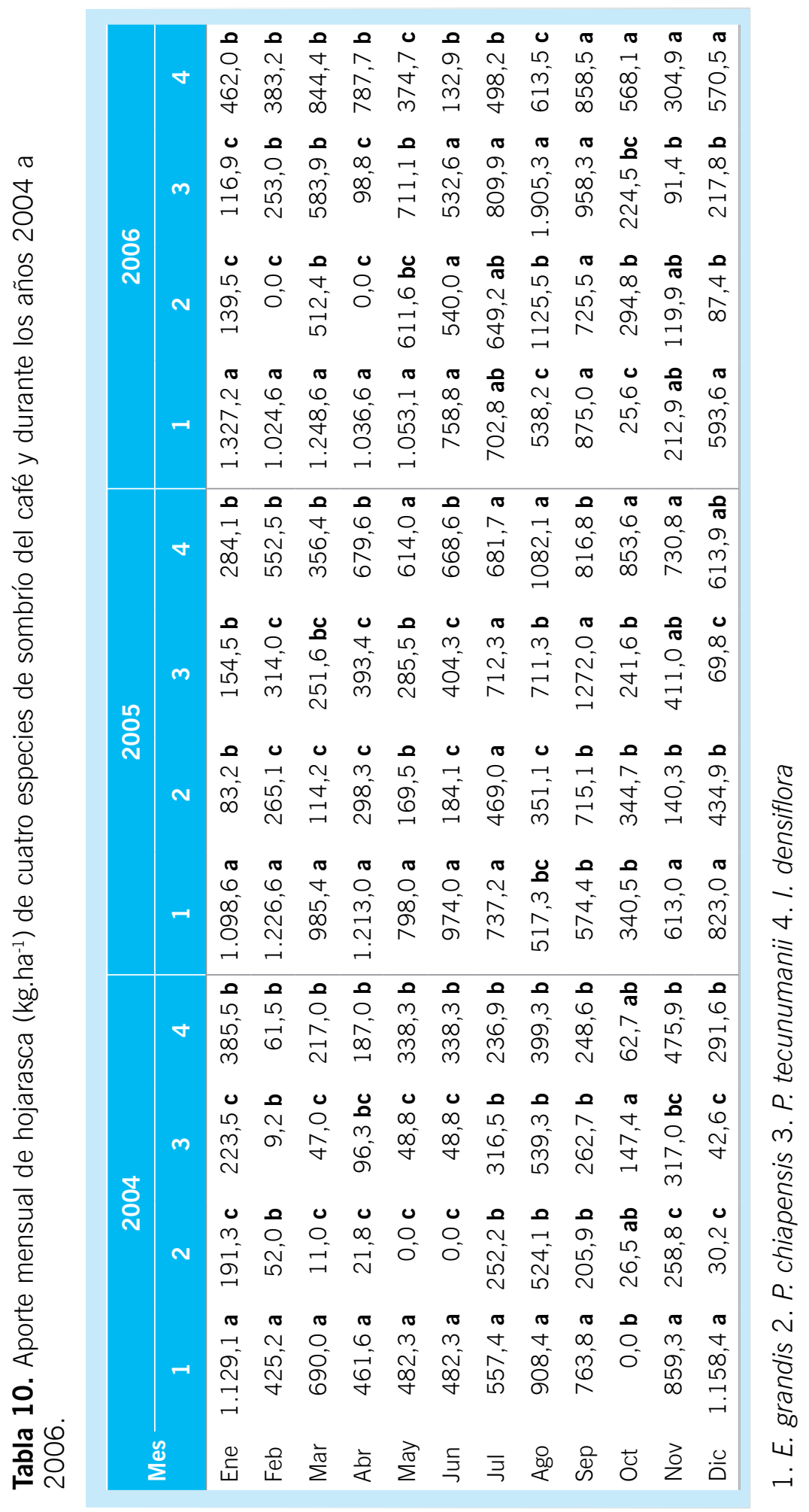


situación coincidente con la deficiencia hídrica registrada en el mes de agosto (Figura 9); este comportamiento de $E$. grandis también se presentó en el año 2004. P. chiapensis tuvo su mayor producción de biomasa seca entre agosto y septiembre, lapso en el cual aportó el 38,5\% (1.851,0 kg.ha-1) de la biomasa seca total producida, con deficiencias hídricas en el mes de agosto; en los meses de febrero y abril este pino empleado como sombrío del café, no produjo hojarasca, sin embargo esta respuesta no tuvo relación con alguna deficiencia hídrica (Figura 9).

P. tecunumanii produjo su mayor cantidad de biomasa seca $\left(3.673,5 \mathrm{~kg}^{-h^{-1}}\right)$ equivalente al 56,5\% del total, entre los meses de julio a septiembre, el déficit hídrico se registró en agosto; se presentó una producción muy baja de hojarasca en el mes de octubre $(3,5 \%)$ y entre los meses de enero, abril y noviembre $(4,7 \%)$. I. densiflora aportó su mayor cantidad de hojarasca en los meses de marzo y septiembre, esta producción fue del 26,6\% (1.702,9 kg.ha-1) de la biomasa seca total producida, mientras que la producción más baja de hojarasca fue de $132,9 \mathrm{~kg} \cdot \mathrm{mes}^{-1}$ y se registró en el mes de junio. Schaffers (58) encontró que el contenido de agua del suelo es el factor que más incide en la cantidad y calidad de la biomasa seca aportada por los árboles en comunidades de plantas.

Las mayores producciones de biomasa seca durante las tres evaluaciones las registró $E$. grandis, éstas fueron de 7.918; 9.901 y $9.397 \mathrm{~kg}$ ha $^{-1}$ en los años 2004, 2005 y 2006, respectivamente; el promedio de la producción de hojarasca fue de $9.072 \mathrm{~kg}$.ha-1. año-1. Las producciones de hojarasca más bajas se registraron en $P$. Chiapensis y fueron de $1.574 ; 3.569$ y $4.806 \mathrm{~kg}$ ha $^{-1}$ en los años 2004,2005 y 2006, respectivamente, con un promedio de la producción de hojarasca de $3.316 \mathrm{~kg}$ ha- ${ }^{-1}$ año-1. . P. tecunumanii e I. densiflora produjeron en promedio 5.512 y 4.954 kg.ha- ${ }^{-1}$.año-1 de hojarasca, respectivamente.

Eucalyptus salubris puede producir cerca de $1.169 \mathrm{~kg} \cdot \mathrm{ha}^{-1}$.año-1 de materia seca, Herwitz y Gutterman (28) y Maggs (41) indican que el promedio de la producción anual de biomasa seca producida por Pinus elliottii puede ser de 6.5 t.ha-1. . En plantaciones de coníferas compuestas por Pinus pinaster, Pinus nigra y Abies borisii, Kavvadias et al. (31)

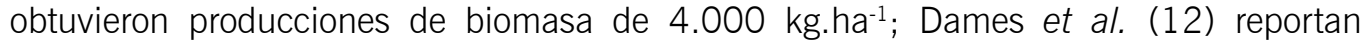
producciones de biomasa seca entre 3,64 y 5,89 t.ha- ${ }^{-1}$.año-1 en Pinus patula. Estudios realizados por Urrego y Farfán (70), muestran que el aporte de materia seca media al año en nogal, pino y eucalipto fue de 3,5; 6,7 y 6,4 t.ha-1 año-1 $^{-1}$. Segura et al. (60), estimaron que la biomasa de los árboles Cordia alliodora, Juglans olanchana, Inga tonduzzi e I. punctata empleados como sombrío del café, oscila entre 3,5 y 886 kg por árbol.

Nolte et al. (47) en Calliandra calothyrsus (3.947 plantas/ha), registraron producciones entre 18,9 y $25,0 \mathrm{Mg}$. ha- ${ }^{-1}$ de materia seca durante dos años, y en Acacia senegal, Deans et al. (14), reportan producciones de biomasa seca de $1.770 \mathrm{~kg} \cdot \mathrm{ha}^{-1}$.año ${ }^{-1}$ plantados a $6,0 \times 6,0 \mathrm{~m}$. En estudios sobre balances de la materia orgánica en sistemas agroforestales, Fassbender et al. (18), reportan producciones de biomasa seca de 1.10,6 t.ha-1 en Cordia alliodora y de 13,6 t.ha- ${ }^{-1}$.año-1 en Erythrina poeppigiana. 


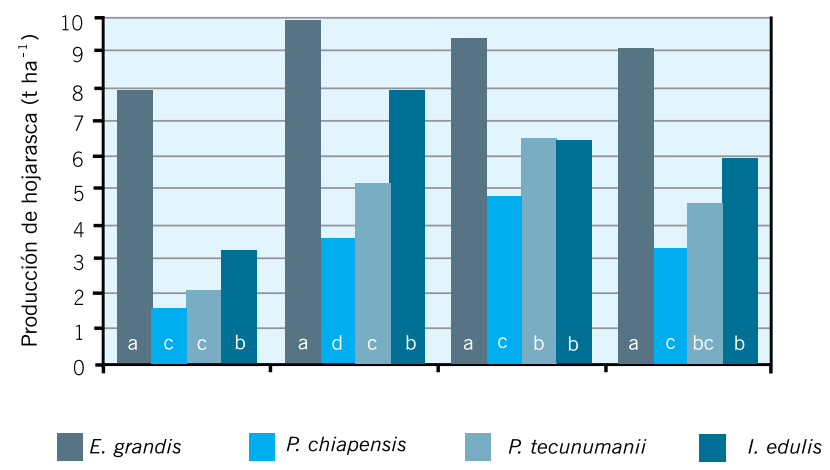

Concentración de nutrientes. Las concentraciones de N, P, K, Ca, Mg y materia orgánica en la hojarasca de las cuatro especies arbóreas, se presentan en la Tabla 11.

La concentración media de $\mathrm{N}$ de la hojarasca producida por $E$. grandis fue de 0,79\%, en P. chiapensis de 0,79\%, en P. tecunimanii de 0,55\% y en I. densiflora del 1,9\%. Los promedios de las concentraciones de $\mathrm{P}$ en la hojarasca producida por estas especies fueron de 0,037\%,0,032\%,0,023\% y 0,057\%, respectivamente.

Los promedios de las concentraciones de $\mathrm{K}$ en la hojarasca producida por $E$. grandis, $P$. chiapensis, $P$. tecunumanii e $I$. densiflora fueron de 0,37\%,0,17\%, 0,16\% y 0,41\%, respectivamente. Y los promedios de las concentraciones de Ca fueron de 1,15\%, 1,30\%, $0,62 \%$ y 0,10\%, para las mismas especies arbóreas.

El promedio de la concentración de $\mathrm{Mg}$ de la hojarasca producida por $E$. grandis fue de $0,15 \%$, en $P$. chiapensis de $0,16 \%$, en $P$. tecunimanii de $0,11 \%$ y en $I$. densiflora del $0,15 \%$. Los promedios de las concentraciones de Materia Orgánica en la hojarasca producida por estas especies fueron de 95,9\%, 94,9\%, 94,7\% y 92,3\%, respectivamente.

El promedio del contenido de $\mathrm{N}$ en la biomasa seca producida por 1 . densiflora fue de 92,7 kg.ha-1, el cual fue significativamente mayor al contenido de $\mathrm{N}$ en la hojarasca producida por $E$. grandis $\left(71,0 \mathrm{~kg} \mathrm{ha}^{-1}\right)$ y al promedio del contenido en la materia seca producida por las dos especies de pino, 26,1 y 28,4 kg.ha ${ }^{-1}$ en $P$. chiapensis y $P$. tecunumanii, respectivamente. Los valores de los contenidos de $\mathrm{N}$ en las dos especies de pino no fueron estadísticamente diferentes (Figura $10 \mathrm{a}$ ). Molinero y Pozo (43) obtuvieron contenidos anuales de $\mathrm{N}$ en la hojarasca de Eucalyptus globulus de $40 \mathrm{~kg}^{\mathrm{h}} \mathrm{ha}^{-1}$. Calliandra calothyrsus establecida a 3.947 plantas/ha, produce cerca de $225 \mathrm{~kg} \mathrm{ha}^{-1}$ de N (47). En Acacia senegal plantado a 6,0 × 6,0 m, Deans et al. (14) afirman que éstos acumulan cerca de 945 g/árbol de N, y Slodicak et al. (23), indican que el N acumulado en la materia seca producida por Picea abies fluctúa entre 720 y 950 kg.ha-1. 
Contenido de nutrientes. Los contenidos de nutrientes en la biomasa seca producida por las cuatro especies arbóreas empleadas como sombrío del café se presentan en la Figura 10.

El promedio del contenido de $\mathrm{P}$ en la hojarasca producida por E. grandis e I. densiflora fueron estadísticamente iguales, con valores de 3,2 kg. ha-1 en el eucalipto y 2,8 kg. ha-1 en el guamo. Los contenidos de $P$ en estas dos especies fueron superiores a los contenidos de las dos especies de pino, que presentaron valores de 1,0 y $1,1 \mathrm{~kg}^{-}$ha ${ }^{-1}$ en $P$. chiapensis y P. tecunumanii, respectivamente (Figura 10 b). Calliandra calothyrsus, establecida a 3.947 plantas/ha, produce cerca de 19 kg.ha- ${ }^{-1}$ de P (47); y en Acacia senegal plantados a 6,0 × 6,0 m, Deans et al. (14) indican que éstos acumulan cerca de $38 \mathrm{~g} / a$ rbol de P.

Los mayores contenidos de $\mathrm{K}$ se registraron en la hojarasca producida por $E$. grandis $\left(33,6 \mathrm{~kg} \mathrm{ha}^{-1}\right)$, los cuales fueron diferentes estadísticamente a los producidos por I. densiflora $\left(9,6 \mathrm{~kg} \cdot \mathrm{ha}^{-1}\right)$. Las dos especies de pino presentaron los más bajos contenidos de $\mathrm{K}$ en la hojarasca, con valores de 6,2 kg.ha- ${ }^{-1}$ en $P$. chiapensis y 9,6 kg.ha-1 en $P$. tecunumanii (Figura $10 \mathrm{c}$ ). En Acacia senegal plantados a 6,0 x 6,0 m, Deans et al. (14) indican que éstos acumulan cerca de 420 g/árbol de K.

Tabla 11. Concentraciones (\%) de nutrientes de la hojarasca de cuatro especies de sombra del café.

\begin{tabular}{|c|c|c|c|c|c|c|c|c|}
\hline \multirow{2}{*}{ Especies } & \multicolumn{4}{|c|}{ Nitrógeno (\%) } & \multicolumn{4}{|c|}{ Fósforo (\%) } \\
\hline & 2004 & 2005 & 2006 & Media & 2004 & 2005 & 2006 & Media \\
\hline E. grandis & 0,81 & 0,79 & 0,78 & 0,79 & 0,041 & 0,036 & 0,034 & 0,037 \\
\hline P. chiapensis & 0,76 & 0,82 & 0,78 & 0,79 & 0,036 & 0,031 & 0,028 & 0,032 \\
\hline P. tecunumanii & 0,64 & 0,51 & 0,51 & 0,55 & 0,026 & 0,022 & 0,021 & 0,023 \\
\hline I. densiflora & 1,90 & 1,95 & 1,85 & 1,90 & 0,055 & 0,054 & 0,061 & 0,057 \\
\hline \multirow{2}{*}{ Especies } & \multicolumn{4}{|c|}{ Potasio (\%) } & \multicolumn{4}{|c|}{ Calcio (\%) } \\
\hline & 2004 & 2005 & 2006 & Media & 2004 & 2005 & 2006 & Media \\
\hline E. grandis & 0,37 & 0,37 & 0,36 & 0,37 & 1,18 & 1,17 & 1,09 & 1,15 \\
\hline P. chiapensis & 0,16 & 0,18 & 0,16 & 0,17 & 1,15 & 1,35 & 1,39 & 1,30 \\
\hline P. tecunumanii & 0,16 & 0,16 & 0,17 & 0,16 & 0,65 & 0,62 & 0,58 & 0,62 \\
\hline I. densiflora & 0,36 & 0,46 & 0,42 & 0,41 & 0,12 & 0,09 & 0,08 & 0,10 \\
\hline \multirow{2}{*}{ Especies } & \multicolumn{4}{|c|}{ Magnesio (\%) } & \multicolumn{4}{|c|}{ Materia orgánica (\%) } \\
\hline & 2004 & 2005 & 2006 & Media & 2004 & 2005 & 2006 & Media \\
\hline E. grandis & 0,15 & 0,17 & 0,13 & 0,15 & 95,8 & 95,9 & 96,0 & 95,9 \\
\hline P. chiapensis & 0,15 & 0,17 & 0,16 & 0,16 & 94,8 & 94,6 & 95,2 & 94,9 \\
\hline P. tecunumanii & 0,13 & 0,11 & 0,10 & 0,11 & 93,9 & 94,9 & 95,2 & 94,7 \\
\hline I. densiflora & 0,17 & 0,15 & 0,13 & 0,15 & 91,7 & 92,6 & 92,7 & 92,3 \\
\hline
\end{tabular}


El promedio del contenido de Ca en la biomasa seca producida por E. grandis fue de 106,1 kg. ha ${ }^{-1}$, el cual fue significativamente mayor al contenido de Ca en la hojarasca producida por I. densiflora $\left(4,5 \mathrm{~kg} \cdot \mathrm{ha}^{-1}\right)$ y al promedio del contenido en la materia seca producida por las dos especies de pino, que presentaron valores de 43,0 y 31,6 $\mathrm{kg}^{\text {. ha-1 }}{ }^{-1}$ en $P$. chiapensis y $P$. tecunumanii, respectivamente; cabe anotar que el contenido de $\mathrm{Ca}$ en las dos especies de pino no fue estadísticamente diferente (Figura $10 \mathrm{~d}$ ). I. densiflora en asociaciones con café como sombrío y establecido a 12,0 x 12,0 m, puede aportar cerca de 11,0 t.ha $^{-1}$.año-1 de materia seca, 199,0 kg de N, 7,7 kg de P, 48,9 kg de K, $158 \mathrm{~kg}$ de Ca y 27,3 kg.ha-1.año-1 de Mg (8).

Los mayores contenidos de Mg los presentó la hojarasca producida por E. grandis (14,9 $\mathrm{kg} \cdot \mathrm{ha}^{-1}$ ), los cuales fueron diferentes estadísticamente a los producidos por I. densiflora
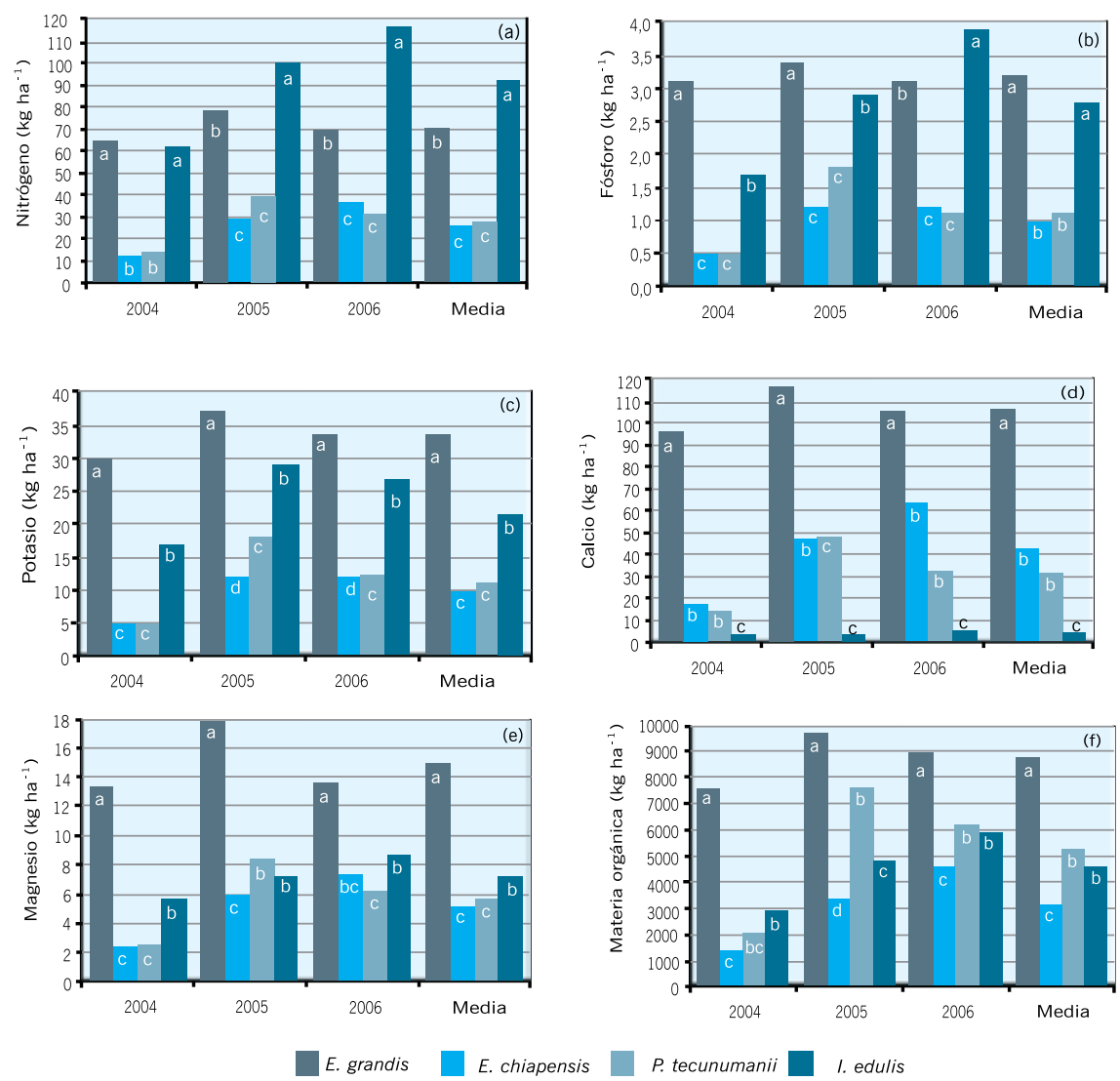

Figura 10. Contenido de (a) Nitrógeno, (b) Fósforo, (c) Potasio, (d) Calcio, (e) Magnesio y (f) Materia Orgánica, en la hojarasca producida por E. grandis, P. chiapensis, P. tecunumanii e I. densiflora. 
(7,2 kg.ha-1); las dos especies de pino presentaron los más bajos contenidos de Mg en la hojarasca, 5,2 kg.ha-1 en $P$. chiapensis y 5,7 kg. ha-1 en $P$. tecunumanii (Figura 10 e).

Leclau et al. (35) encontraron que la biomasa producida por Eucalyptus sp., hasta de siete años de edad contenía $100 \mathrm{~kg}$ de N, 30 kg de P, 39 kg de K, 33 kg de Ca y 19 kg de Mg por hectárea, y Maggs (41) obtuvo que la biomasa seca producida por Pinus elliottii puede contener cerca de 22,8 kg.ha ${ }^{-1}$ de N, 1,5 kg.ha-1 de P, 4,5 kg.ha ${ }^{-1}$ de $\mathrm{K}$, 33,9 kg.ha ${ }^{-1}$ de Ca y 9,3 kg.ha ${ }^{-1}$ de Mg. En plantaciones de coníferas compuestas por Pinus pinaster, Pinus nigra, y Abies borisii, Kavvadias et al. (31) obtuvieron que los contenidos de nutrientes en la biomasas seca fluctuaban entre 235,4 y $913,9 \mathrm{~kg}$. ha-1 de $\mathrm{N}, 15,4$ y 55,8 kg.ha ${ }^{-1}$ de P; 40,4 y 113,1 kg.ha-1 de K; 99,3 y 506,9 kg.ha-1 de Ca y 39,9 a 285,5 kg.ha-1 de Mg.

Los promedios de los contenidos de Materia Orgánica en la biomasa seca producida por $E$. grandis fueron de $8.745 \mathrm{~kg}$.ha-1 ${ }^{-1}$ el cual fue significativamente mayor al contenido de

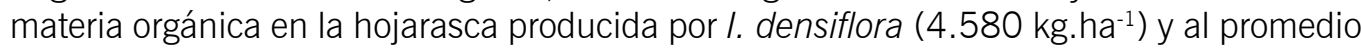
del contenido en la materia seca producida por las dos especies de pino, con valores de 3.136 y $5.256 \mathrm{~kg}$ ha-1 ${ }^{-1}$ en $P$. chiapensis y P. tecunumanii, respectivamente; el contenido de materia orgánica en las dos especies de pino fue estadísticamente diferente (Figura 10 f).

De este estudio se estableció que:

- El promedio de la producción de hojarasca de E. grandis, en los tres períodos de evaluación, fue de $9.072 \mathrm{~kg}$.ha- ${ }^{-1}$.año ${ }^{-1}$, en $P$. chiapensis fue de $3.316 \mathrm{~kg}$.ha- ${ }^{-1}$, y para las especies $P$. tecunumanii e $I$. densiflora los promedios fueron de 5.512 y 4.954 $\mathrm{kg} \cdot \mathrm{ha}^{-1} \cdot \mathrm{año} \mathrm{O}^{-1}$.

- El promedio del contenido de $\mathrm{N}$ en la biomasa seca producida por I. densiflora fue de $92,7 \mathrm{~kg}$ ha-1 ${ }^{-1}$. E. grandis tuvo $71,0 \mathrm{~kg}$ de $\mathrm{N}$ ha ${ }^{-1}$ y en $P$. chiapensis y $P$. tecunumanii los contenidos fueron de 26,1 y $28,4 \mathrm{~kg}$. ha ${ }^{-1}$.

- Los promedios del contenido de $\mathrm{P}$ en la hojarasca producida por E. grandis e I. densiflora fueron de 3,2 y $2,8 \mathrm{~kg} \cdot \mathrm{ha}^{-1}$, y en $P$. chiapensis y $P$. tecunumanii los valores fueron de 1,0 y $1,1 \mathrm{~kg}$.ha-1, respectivamente.

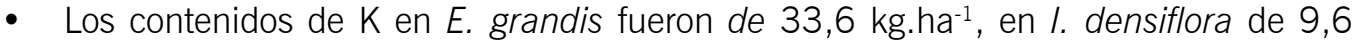
kg.ha-1, en $P$. chiapensis de 6,2 kg.ha-1 y de 9,6 kg. ha-1 en $P$. tecunumanii.

- El promedio del contenido de Ca en la biomasa seca producida por E. grandis fue de 106,1 kg.ha-1 ${ }^{-1}$ en I. densiflora de 4,5 kg.ha- ${ }^{-1}$, y de 43,0 y 31,6 kg.ha ${ }^{-1}$ en $P$. chiapensis y $P$. tecunumanii, respectivamente.

- Los promedios de los contenidos de Materia Orgánica en la biomasa seca producida por E. grandis fueron de $8.745 \mathrm{~kg}$.ha- ${ }^{-1}$, en $I$. densiflora de $4.580 \mathrm{~kg}^{-h^{-1}}$ y de 3.136

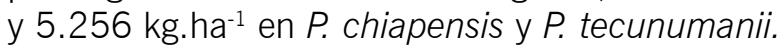




\section{LITERATURA CITADA}

1. ARCILA P., J.; JARAMILLO R., A. Relación entre la humedad del suelo, la floración y el desarrollo del fruto del cafeto. Avances Técnicos Cenicafé (Colombia) No. 311:1-8. 2003.

2. ARGÜELLO A., H. Cambios en la composición bioquímica y su aplicabilidad en el uso de follajes verdes como fuente de materia orgánica y nutrimentos en sistemas agroforestales. Agronomía Colombiana (Colombia) 12(2):169-181. 1995.

3. AUSTIN A.T.; VITOUSEK P.M. Precipitation, decomposition and litter decomposability of Metrosideros polymorpha in native forests on Hawaii. Journal of Ecology, 88(1):129-138. 2000.

4. BERG, B. Litter decomposition and organic matter turnover in northern forest soils, Forest Ecology and Management, 133(2000):13-22. 2000.

5. BUNVONG, T.; O, GRANGER. El clima y la descomposición de la hojarasca en el bosque tropical. Agri-silvicultura en América Latina - FAO. Unasylva, 31(126):45-56. 1979.

6. CALLAhAm, M. A.; ANDERSON, P., H.; WALDROP, T., A.; LIONE, D., J.; SHELBURNE, V., B. Litter decomposition and soil respiration responses to fuel-reduction treatments in Piedmont loblolly pine forests. In: K. Connor, ed., Proceedings, 12th Biennial Southern Silvicultural Research Conference, Biloxi, MS. Gen. Tech. Rep. SRS-71. Asheville, NC: U.S. Department of Agriculture, Forest Service, Southern Research Station. 2004. p. 25-29.

7. CAMIRÉ C.; TROFYMOW J. A.; DUSCHENE L.; MOORE T.R.; KOZAK L.; TITUS B.; KRANABETTER M.; PRESCOTT C.; VISSER S.; MORRISON I.; SILTANEN M.; SMITH S.; FYLES J.; WEIN R. Rates of litter decomposition over 6 years in Canadian forests: Influence of litter quality and climate. Canadian Journal of Forest Research. 32(5):789-804. 2002.

8. CARDONA C., D.A.; SADEGHIAN K., S. Aporte de material orgánico y nutrientes en cafetales al sol y bajo sombrío de guamo. Avances Técnicos Cenicafé (Colombia) No. 334:1-8. 2005.

9. CENTRO NACIONAL DE INVESTIGACIONES DE CAFÉ - CENICAFÉ; Disciplina de Agroclimatología. Archivos de información climática hasta 1998. Chinchiná (Colombia).

10. CENTRO NACIONAL DE INVESTIGACIONES DE CAFÉ - Cenicafé. CHINCHINÁ. COLOMBIA. Anuario Meteorológico Cafetero 2004. Chinchiná (Colombia), Cenicafé, 2005. p. 474.

11. CORBEELS M. Plant Litter and Decomposition: General Concepts and Model Approaches. CSIRO Forestry and Forest Products. Neework shop proceedings, 18-20:124-129. 2001.

12. DAMES, J. F.; SCHOLES, M. C.; STRAKER, C. J. Litter production and accumulation in Pinus patula plantations of the Mpumalanga Province, South Africa. Plant and Soil, 203:183-190. 1998.

13. DE LAS SALAS., G. Suelos y ecosistemas forestales con énfasis en América tropical. San José (Costa Rica), IICA, 1987. 450 p.

14. DEANS J. D.; DIAGNE O.; LINDLEY D. K.; DIONE M.; PARKINSON J. A. Nutrient and organicmatter accumulation in Acacia senegal fallows over 18 years. Forest Ecology and Management, 124(2-3):153-167. 1999. 
15. DORA, M.; VILLELA, D. M.; PROCTOR, J. Leaf Litter Decomposition and Monodominance in the Peltogyne Forest of Maracá Island, Brazil. Biotropica, 34(3): 334-347. 2002.

16. FASSBENDER, H.W.; BORNEMISZA, E. Química de suelos, con énfasis en suelos de América Latina. Colección Libros y Materiales Educativos IICA, No 81. 1987. 420 p.

17. FASSBENDER, H.W. Modelos edafológicos de sistemas agroforestales. 2a. Ed. Centro Agronómico Tropical de Investigación y Enseñanza, Proyecto Agroforestal CATIE-GTZ, Turrialba. 1987. 491 p.

18. FASSBENDER, H. W.; BEER, J.; HEUVELDOP, J.; IMBACH, A.; ENRIQUEZ, G.; BONNEMANN, A. Ten year balances of organic matter and nutrients in agroforestry systems at CATIE, Costa Rica. Forest Ecology and Management, 45(1-4):173-183. 1991.

19. FEDERACIÓN NACIONAL DE CAFETEROS DE COLOMBIA. BOGOTÁ. COLOMBIA. Caracterización edáfica del Ecotopo 211A. Santa Fé de Bogotá (Colombia), FEDERACAFÉ, 1998.

20. FEDERACIÓN NACIONAL DE CAFETEROS - FNC.; MINISTERIO DEL MEDIO AMBIENTE MINAMBIENTE. BOGOTÁ. COLOMBIA. Estudio descriptivo de suelos, Lote Suecia, Finca La Suecia, Municipio El Tambo, Departamento del Cauca. Bogotá (Colombia), FNC, 2001. 58 p.

21. FLORENCE R.G. Ecology and silviculture of eucalypt forests. CSIRO Publishing, Australia, 1996. $414 \mathrm{p}$.

22. GAMA-RODRIGUES, A. C.; BARROS, N. F.; SANTOS, M. Decomposição e liberação de nutrientes do folhedo de espécies florestais nativas em plantios puros e mistos no sudeste da bahia. Revista Brasileira de Ciencia do Solo, 27(6):1021-1031. 2003.

23. GANJEGUNTE G. K.; CONDRON L. M.; CLINTON P. W.; DAVIS M. R.; MAHIEU N. Decomposition and nutrient release from radiata pine (Pinus radiata) coarse woody debris. Forest Ecology and Management, 187(2):197-211. 2004.

24. GÓMEZ G., L.; JARAMILLO R., A. El clima de la zona cafetera colombiana y su relación con el cultivo del café. In: 50 Años de Cenicafé 1938-1988. Conferencias Conmemorativas. Chinchiná (Colombia), CENICAFÉ, 1990. p. 23-32.

25. GUO L.B.; SIMS R.E.H. Effects of light, temperature, water and meatworks effluent irrigation on eucalypt leaf litter decomposition under controlled environmental conditions. Applied Soil Ecology, 17(3):229-237. 2001.

26. HARTEMINK, A. E.; O'SULLIVAN, J. N. Leaf litter decomposition of Piper aduncum, Gliricidia sepium and Imperata cylindrica in the humid lowlands of Papua New Guinea. Plant and Soil, 230:115-124, 2001.

27. HENIN, S.; DUPUIS, M. Essai de bilan de la matière organique des sols. Annual. Agronomy, 15(1):161-172. 1945.

28. HERWITZ, S. R.; GUTTERMAN, Y. Biomass production and transpiration efficiencies of eucalyptus in the Negev Desert. Forest Ecology and Management, 31(1-2):81-90. 1990. 
29. HEUVELDOP, J.; ALPIZAR O., L. A.; FASSBENDER, H.W.; ENRIQUEZ C., G.A.; FOLSTER, H. Modeling agroforestry systems of cacao (Theobroma cacao) with laurel (Cordia alliodora) and poro (Erythrina poeppigiana) in Costa Rica. II. Cacao and wood production, litter production and decomposition. Agroforestry Systems, 6:37-48. 1989.

30. JARAMILLO R., A. Climatología de región andina de Colombia; microclima y fenología del cultivo del café. Chinchiná (Colombia), CENICAFÉ, 2000. 172 p.

31. KAVVADIAS, V. A.; ALIFRAGIS, D.; TSIONTSIS, A.; BROFAS, G.; STAMATELOS, G. Litterfall, litter accumulation and litter decomposition rates in four forest ecosystems in northern Greece. Forest Ecology and Management, 144(1-3):113-127. 2001.

32. KOCHY, M.; SCOTT D. W. Litter decomposition and nitrogen dynamics in aspen forest and mixedgrass prairie. Ecology, 78(3):732-739. 1997.

33. KWABIAH A. B.; VORONEY R. P.; PALM C.A.; N.C. STOSKOPF. Inorganic fertilizer enrichment of soil: effect on decomposition of plant litter under subhumid tropical conditions. Biology and Fertility of Soils, 30(3):224-231. 1999.

34. LABRADOR M., J. Materia orgánica en los agrosistemas. Madrid. Ministerio de agricultura, Pesca y Alimentación: Mundi-Prensa, D.L. 1996. 174 p.

35. LACLAU, J. P.; BOUILLET, J. P.; RANGER, J. Dynamics of biomass and nutrient accumulation in a clonal plantation of Eucalyptus in Congo. Forest Ecology and Management, 128(3):181-196. 2000.

36. LISKI J.; NISSINEN A.; ERHARD M.; TASKINEN O. Climatic effects on litter decomposition from arctic tundra to tropical rainforest. Global Change Biology, 9(4):575-584. 2003.

37. LÓPEZ, A. M. Sistemas agroforestales tipo multiestratos. Asociación Latinoamericana de Integración (ALADI), Departamento de Promoción Económica Montevideo, Publicación No. $11 / 01$ abril de 2001. $50 \mathrm{p}$

38. LUGO, A. E.; WANG, D.; BORMANN, F. H. A comparative analysis of biomass production in five tropical tree species. Forest Ecology and Management, 31(3):153-166. 1990.

39. LUPWAYI N. Z.; HAQUE I. Leucaena hedgerow intercropping and cattle manure application in the Ethiopian highlands. I. Decomposition and nutrient release. Biology and Fertility of Soils, 28(2):182-195. 1998.

40. LUSK C. H.; DONOSO C.; JIMÉNEZ M.; MOYA C.; OYARCE G.; REINOSO R.; SALDAÑA A.; VILLEGAS P.; MATUS F. Decomposition of leaf litter of Pinus radiata and three native tree species. Revista Chilena de Historia Natural, 74:705-710, 2001.

41. MAGGS, J. Litter fall and retranslocation of nutrients in a refertilized and prescribed burned Pinus elliottii plantation. Forest Ecology and Management, 12(3-4):253-268. 1985.

42. MCTIERNAN K.B.; COUTEAUX M.-M.; BERG B.; BERG M.P.; CALVO DE ANTA R.; GALLARDO A.; KRATZ W.; PIUSSI P.; REMACLE J.; VIRZO DE SANTO A. Changes in chemical composition of Pinus sy/vestris needle litter during decomposition along a European coniferous forest climatic transect Soil. Biology and Biochemistry, 35(6):801-812. 2003. 
43. MOLINERO., J.; POZO., J. Impact of a eucalyptus (Eucalyptus g/obulus Labill.) plantation on the nutrient content and dynamics of coarse particulate organic matter (CPOM) in a small stream Hydrobiologia, 528:143-165. 2004.

44. MONTAGNINI, F. RAMSTAD, K. SANCHO, M. F. Litterfall, litter decomposition and the use of mulch of four indigenous tree species in the Atlantic lowlands of Costa Rica. Agroforestry Systems, 23(1):39-61. 1993.

45. MORO M., A. J.; DOMINGO F. Litter Decomposition in Four Woody Species in a Mediterranean Climate: Weight Loss, N and P Dynamics. Annals of Botany, 86:1065-1071, 2000.

46. MUNGUÍA., R.; BEER., J.; HARMAND., J-M.; HAGGAR., J. Tasas de descomposición y liberación de nutrientes de la hojarasca de Eucalyptus deg/upta, Coffea arabica y hojas verdes de Erythrina poeppigiana, solas y en mezclas. Agroforestería en las Américas, № 41- 42. 2004.

47. NOLTE., C.; TIKI-MANGA., T.; BADJEL-BADJEL. S.; GOCKOWSKI., J.; HAUSER., S.; WEISE S. F. Effects of calliandra planting pattern on biomass production and nutrient accumulation in planted fallows of southern Cameroon. Forest Ecology and Management, 179(1-3):535-545. 2003.

48. OLIVER L.; PÉREZ C., M. E.; BERMúDEZ DE C., F. Degradación de la hojarasca de un pastizal oligotrófico mediterráneo del centro de la Península Ibérica. Anales de Biología, 24:21-32. 2002.

49. OELBERMANN M.; VORONEY R. P.; SCHLÖNVOIGT A. M.; KASS D. C. L. Decomposition of Erythrina poeppigiana leaves in 3, 9, and 18 year-old alleycropping systems in Costa Rica. Agroforestry Systems (Holanda), 63:27-32. 2004.

50. OLSON, J.S. Energy storage and the balance of producers and decomposers in ecological systems. Ecology, 44:322-331. 1963.

51. PARKASH T., O.; KUMAR., P.; KHOSLA P., K. Structure and function of traditional agroforestry systems in the western Himalaya. I. Biomass and productivity. Agroforestry Systems, 9:47-70. 1989.

52. PRAUSE, J; LIFSCHITZ, A. P. Constantes de descomposición de hojas y la relación lignina/ celulosa en cuatro especies forestales de la Reserva Estricta de Colonia Benítez - Chaco. Comunicaciones científicas y tecnologicas 2001. Universidad Nacional del Nordeste, Argentina. On-line Internet: www.unne.edu.ar/cyt/2001/cyt.htm (consultado enero de 2007).

53. PURI, S.; SINGH, V.; BHUSHAN, B.; SINGH, S. Biomass production and distribution of roots in three stands of Populus deltoides. Forest Ecology and Management, 65(2-3):135-147. 1994.

54. RIBEIRO C.; MADEIRA M.; ARAUJO M.C. Decomposition and nutrient release from leaf litter of Eucalyptus globulus grown under different water and nutrient regimes. Forest Ecology and Management, 171(1):31-41. 2002.

55. SANTA, R. I. Litter fall, decomposition and nutrient release in three semi-arid forests of the Duero basin, Spain. Forestry, 74(4):347-358. 2001.

56. SAÑA J. Mineralització de la Fracció Organiconitrogenada dels Adobs Orgànics: Possibles Vies Per A La Seva. Estimació Dossiers Agraris, Problemes moderns en l'ús dels sòls: nitrats. Institució Catalana D'estudis Agraris - ICEA, 1999. p 29-40 
57. SARIYILDIZ, T. J. Litter Decomposition of Picea orientalis, Pinus sy/vestris and Castanea sativa Trees Grown in Artvin in Relation to Their Initial Litter Quality Variables. Turkish journal of agriculture and forestry, (27):237-243. 2003.

58. SCHAFFERS A., E. P. Soil, biomass, and management of semi-natural vegetation Part I. Interrelationships. Plant Ecology, 158:229-246. 2002.

59. SCHROTH G.; LEHMANN J.; RODRIGUES M. R. L.; BARROS E.; MACÊDO J. L. V. Plant-soil interactions in multistrata agroforestry in the humid tropicsa. Agroforestry Systems, 53(2):85102. 2001.

60. SEGURA., M.; KANNINEN., M.; SUÁREZ., D. Allometric models for estimating aboveground biomass of shade trees and coffee bushes grown together. Agroforestry Systems, 68:143-150. 2006.

61. SIMMONS, T. T.; HAWKINS, C.P. Factors influencing lotic leaf litter decomposition rates: results of a large scale study in Western streams, Presented at the NABS Annual meeting, Vancouver, British Columbia, in Organic Matter Processing. North American Benthological Society. 2004. On-line internet: www. benthos.org/database/allnabstracts.cfm/db/Vancouver2004abstracts/ id/201 (consultado septiembre de 2005)

62. SINGH K. P.; SINGH P. K.; TRIPATHI S. K. Litterfall, litter decomposition and nutrient release patterns in four native tree species raised on coal mine spoil at Singrauli, India. Biology and Fertility of Soils, 29:371-378. 1999

63. SINHA, M. K.; SINHA, D. P.; SINHA, H. Organic matter transformations in soils (V): kinetics of carbon and nitrogen mineralization in soils amended with diferent organic materials. Plant and Soils, 46:579-590. 1977.

64. SLODICAK, M.; NOVAK, J.; SKOVSGAARD, J. P. Wood production, litter fall and humus accumulation in a Czech thinning experiment in Norway spruce (Picea abies (L.) Karst.). Forest Ecology and Management, 209(1-2):157-166. 2005.

65. SNOWDON, P.; BENSON, M. L. Effects of combinations of irrigation and fertilization on the growth and above-ground biomass production of Pinus radiata. Forest Ecology and Management, 52(14):87-116. 1992.

66. STAVER C.; GUHARAY F.; MONTERROSO D.; MUSCHLER R. G. Designing pest-suppressive multistrata perennial crop systems: shade-grown coffee in Central America. Agroforestry Systems, 53(2):151-170. 2001.

67. STIGTER C. J.; MOHAMMED A. E.; NASR AL-AMIN N. K.; ONYEWOTU L. O. Z.; OTENG'I S. B. B.; KAINKWA R. M. R. Agroforestry solutions to some African wind problems. Journal of Wind Engineering and Industrial Aerodynamics, 90(10):1101-1114. 2002.

68. THAIUTSA, B.; GRANGER, O. El clima y la descomposición de la hojarasca en el bosque tropical. Unasylva, 31(126):121-129. 1979

69. TUNDIS V., A. R.; GUERRINI, I., AMARAL.; FRANKEN, W., KARL.; FONSECA. R, C. B. Produção de serapilheira e ciclagem de nutrientes de uma floresta estacional semidecidual em zona ripária. Revista Árvore, Viçosa-MG. 28(6):793-800, 2004 
70. URREGO, B.; FARFÁN V., F. Aportes de hojarasca en cafetales con sombrío de especies forestales. Cali (Colombia), Smurfit. Cartón de Colombia, 2002. (Informe de Investigación No. 188).

71. WHITE, D. L.; HAINES, B. L.; BORING, L. R. Litter decomposition in southern Appalachian black locust and pine-hardwood stands: litter quality and nitrogen dynamics. Canadian Journal Forest Research, 18I(1I):54-63. 1988

72. WIEDER, R.K.; LANG, G.E. A critique of the analytical methods used in examining decomposition data obtained from litter bags. Ecology, 6(36):1636-1642. 1982.

73. XU X.; HIRATA E.; ENOKI T.; TOKASHIKI Y. Leaf litter decomposition and nutrient dynamics in a subtropical forest after typhoon disturbance. Vegetatio, 173(2):161-170. 2004.

74. ZAIA F. C.; GAMA-RODRIGUES A. C. Ciclagem e balanço de nutrientes em Povoamentos de eucalipto na região Norte fluminense. Seção IV - Fertilidade E nutrição do solo. Revista Brasileira de Ciência do Solo, 28:843-852. 2004.

75. ZHI-AN LI; SHAO-LIN PENG; DEBBIE J. RAE; GUO-YI ZHOU. Litter decomposition and nitrogen mineralization of soils in subtropical plantation forests of southern China, with special attention to comparisons between legumes and non-legumes. Plant and Soil, 229(1):105116. 2001. 